\title{
NASAL INTERNAL AND EXTERNAL AERODYNAMICS FOR HEALTHY AND BLOCKED CAVITIES
}

\author{
SHAHRZAD NAYEBOSSADRI ${ }^{1}$, ELDAD J AVITAL2 ${ }^{2 *}$, FARIBOZ MOTALLEBI ${ }^{3}$, GUY KENYON ${ }^{4}$ \\ ${ }^{1,2,3}$ School of Engineering and Materials Science, Queen Mary University of London \\ 327 Mile End Road London E1 4NS UK \\ ${ }^{4}$ BMI The London Independent Hospital, 1 Beaumont Square London E1 4NL UK \\ emails: shahrzad98@hotmail.com², e.avital@qmul.ac.uk²* \\ f.motallebi@qmul.ac.uk³,'guyskenyon@gmail.com ${ }^{4}$
}

\begin{abstract}
:
Human nasal airflow in a healthy and partially blocked cavities is investigated using computational and experimental means. While previous studies focused on flow inside the nasal cavity, this study also looks at the external air stream coming out of the nostrils. The aim is to investigate the airflow subject to partial blocking in the nasal cavity and assess the potential of using a flow visualization method to identify abnormal nasal geometry. Two methods of study are used; Computational Fluid Dynamics (CFD) and experimental based on Particle Image Velocimetry (PIV). Nasal cavity geometry is reconstructed from CT scans. The flow visualization Schileren method is also demonstrated.

The computational results agree well with previous results in terms of nasal resistance and character of the internal flow. Good agreement is also found in the external aerodynamics during expiration between the computational and experimental results. Several generic partial blockages are investigated to show changes in nasal resistance, turbulence energy and the air-stream leaving the nostrils during expiration. Anterior blockages are found to have more profound effects on all these three aspects, but all show effect on the external air-stream. A possible universal angle for the external air-stream emitted by a healthy nasal cavity is discussed.
\end{abstract}

\section{Keywords:}

Nasal aerodynamics, partial blockage, CFD, PIV, external flow

* Corresponding author 


\section{Introduction:}

The internal human nose or the nasal cavity as it is often called performs a variety of important physiological functions by being the main airway for normal breathing, conditioning the air temperature and humidity, and serving the sense of smell (olfaction). It can also be used for drug delivery. Improper function of the nasal cavity can have a significant adverse effect on individual's quality of life. Thus much attention has been given to the study of the airflow through the nasal cavity using experimental and recently computational means, e.g. Wen et al. ${ }^{1}$ and Brucker et al. ${ }^{2}$.

The nasal cavity is connected at front to the atmosphere through the nostrils and to the pharynx at the back of the cavity. The cavity is divided to left and right air passages that are separated by the nasal septum. Each passage is capable of operating independently from each other and each has a nasal vestibule, a nasal valve, turbinated airways and is connected at the back to the nasopharynx, see Fig 1 for an illustration of the turbinated airways. Early experimental studies concentrated on measuring the variation of the flow rate through the cavity against the pressure difference over it $^{1}$. This resulted in the introduction of the important concept of nasal resistance (NR) which is the ratio between the pressure difference over the cavity to its flow rate.

Two regions of importance were identified for a healthy nasal cavity; low nasal resistance associated with low flow rate and high nasal resistance associated with high flow rate. The first region is associated with mainly laminar flow inside the cavity and the second region with the occurrence of turbulence. The transition between the two regions is around flow rates between $150 \mathrm{ml} / \mathrm{s}$ to $250 \mathrm{ml} / \mathrm{s}$ per passage (left or right) when measured for steady flow. The onset of turbulence was found to occur around the nasal valve, which is a narrowing between the nasal vestibule and the turbinated region and thus can experience local adverse pressure effects ${ }^{3}$. Turbulence in the flow was also identified at the upper olfactory region ${ }^{4}$.

This paper is too short to provide a full review of the rich literature on nasal aerodynamics and only studies directly related to the aims and the tools of the current study are noted. The current study aims to investigate the effect of partial nasal blockage on the airflow inside and outside the nasal cavity, and particularly during expiration. The external airflow during expiration is of importance because while nasal blockages can hamper the functionality of the cavity they are not easy to identify. Finding a good relation between the external nasal airflow and internal blockages can assist diagnosis and reduce the need to use uncomfortable examinations procedures. For this purpose we have used two approaches; a numerical investigation based on Computational Fluid Dynamics (CFD) 
using our in-house Lithium code and experimental study using Particle Image Velocimetry (PIV) to identify flow patterns and velocity values. Both approaches complemented each other as a validation tool since the literature reporting both PIV and computational studies sometimes stated conflicting results.

Brucker et al. ${ }^{5}$ was one of the firsts to use two dimensional PIV technique to measure the instantaneous flow field on a 3:1 enlarged scaled model of the nasal fossa. Results showed significant portion of high velocity in the olfactory region due to an inspiratory flow separation anterior to the middle turbinate. The flow was mainly found to pass through the middle and upper airways in inspiration, and lower and middle airways in expiration with a highly irregular and non-uniform pattern just before the turbinated region. This study was limited due to the low resolution of the anatomic data used for model generation. Kelly et al. $^{2}$ studied the flow patterns using PIV on an accurate model of the cavity based on Hookins et al's method ${ }^{6}$. The flow measurement for a non-oscillatory flow rate of $125 \mathrm{ml} / \mathrm{s}$ suggested only a small fraction of the flow in the olfactory region with peak velocities in the nasal valve and along the cavity floor in the inferior airway. Kim et al. ${ }^{7}$ constructed an accurate cast of the nasal cavity and investigated the airflow at respiratory flux of 125 $\mathrm{ml} / \mathrm{s}$. Flow visualisation revealed that the main nasal passageway is the middle airway with highest velocities at the superior airway.

More recently the nasal airflow has been investigated computationally, taking advantage of development in medical imaging and the computational science ${ }^{8}$. Ishikawa et al. ${ }^{9}, 10$ carried out a numerical investigation assuming unsteady and incompressible laminar flow for a complete respiration cycle of $5.2 \mathrm{~s}$ including a $0.1 \mathrm{~s}$ resting phase. Highest and lowest inspiratory velocities were found along the middle meatus and in the superior regions. High vorticity magnitude were recorded at the anterior portion of the nasal cavity close to the middle turbinate and at the posterior regions with sharp velocity gradients. Leeden at al. ${ }^{3}$ carried a numerical study of unsteady incompressible flow field using implicit LES. In this work highest velocities were at the nasal valve region where the flow had an irregular pattern due to a vortex between the nasal valve and the head of the inferior turbinate. Lowest velocities were observed in the inferior and middle meatuses.

Horschler at al. ${ }^{11}$ modelled the nasal airflow using the steady and incompressible laminar assumption. The results found that inspiratory phase to be dependent on the Reynolds number $(\mathrm{Re})$ as an increase in Re was accompanied by generation of vortices. Both Wen at $\mathrm{al}^{4}$ and Wang et $\mathrm{al}^{12}$ reported presence of vortices in the olfactory region when using the steady incompressible assumption although at Wang et al $^{12}$ the vortices appeared at higher flow rates. Grant et al. ${ }^{13}$ studied the flow in an anatomically accurate model of a 
nasal cavity. Steady incompressible Navier stokes equations were solved considering both laminar and turbulent flows. Close agreement between the laminar and the turbulent predictions suggested that the influence of turbulence on the flow was very low, indicating that laminar flow predominates at the considered resting breathing rates. The flow was also found to be dominated by a separation at the posterior end of the nasal floor and a large recirculation zone was formed along the inner walls of the nasopharynx. Kleven et al. ${ }^{14}$ reviewed the use of CFD to study nasal aerodynamics and particularly possible methods of drug delivery, pointing to the importance of capturing anatomically correct geometry of the nasal cavity.

Detailed studies of the internal airflow in a partially blocked cavity have been fewer than those of a healthy cavity. All as far as we are aware, they have looked only at the airflow inside the nasal cavity and not outside as in this study. Septum perforation was numerically assessed for pre- and post- operation condition ${ }^{15}$. Similar approach was also used by Nomura el al. ${ }^{16}$ to show reduction of wall shear stresses due to repair surgery. The approach of using CFD to assess the effects of repair surgery was also pursued by Patel et al. ${ }^{17}$. The effect of septal deviation was numerically studied by Kim et al. ${ }^{18}$ aiming to find correlation between the nasal anatomy and CFD results. Chen et al ${ }^{19}$ computationally investigated the effect of enlarged inferior turbinate and found the peak of turbulence in the nasal valve for a healthy cavity as was also found by Leeden et al. ${ }^{3}$. The enlarged inferior turbinate increased the peak of the insipration velocity and shear stresses. The effect of external nose deformation causing a change in the nostrils was investigated by Grutzenmacher et al. ${ }^{20}$.

Contrary to previous studies which concentrated on the inspiration flow in partly blocked nasal cavities, this study concentrates on the expiration phase as it directly affects the air flow coming out from the nostril. It is aimed to fundamentally assess whether this air stream is affected by abnormal conditions of the nasal cavity and find characteristics of this effect that can form the basis of a future non-invasive and non-radiative assessment based on flow visualization. The methodology of the study is described next, followed by the results' analysis and conclusions.

\section{Methodology}

Two approaches of investigation were used; computational and experimental.

\subsection{Computational methodology}

The computational approach consisted of simulating the incompressible Navier-Stokes equations using our in-house code Lithium. This code has already been successfully used 
for various fluid engineering problems such as open cavity flow ${ }^{21}$ and complex geometry as of sports engineering ${ }^{22}$. It was also successfully used by Leeden et al. ${ }^{3}$ to simulate nasal cavity flow using the implicit large eddy simulation (ILES) approach. The code is based on staggered structured grid formulation and due to the complex geometry of the nasal cavity, a rectangular grid was used where the no-slip wall conditions were captured using a second order ghost point method ${ }^{22,23}$. Grid sensitivity study found the effects of such numerical approximation to be small for flow properties as nasal resistance and flow patterns. The assumption of a rigid wall is commonly used in CFD studies of the nasal cavity $^{3,4,8,13,14}$ and associated experimental studies of enlarged cavities. It much reduces the complexity of the simulation while Quadrio et $\mathrm{al}^{24}$ argued for its effect to be small on the result, although it can be of importance in people who have cartilage weakness, trauma or high abutment of the lateral crus to the pyriform aperture. The computational technology to simulate the flow-structure interaction for bio-fluid applications exists as was done for the human ureter ${ }^{25}$. It requires good knowledge of the wall structural properties and thus is left for a future study.

Semi-implicit time marching of second order and directional split were used to march the momentum equations in time. The implicit time marching was needed because of the very low CFL (Courant-Friedrichs-Lewy) time-step limit caused by some of the narrow airways in the nasal cavity. A second-order upwind TVD (total variation diminishing) scheme with the Van-Leer flux limiter was used to compute the convection terms and a second-order central scheme was used for the diffusion terms. A pent-diagonal matrix solver was used to solve the time-implicit momentum equations during the time marching ${ }^{3}$. Since the primary aim of this study was to look at the effect of the nasal blockage on the airflow, particularly during steady expiration as in medical check, a transitional k- $\omega$ RANS model was added to accelerate the accumulation of the turbulent flow statistics ${ }^{22}$ (if it exists).

A divergence-free velocity field was achieved using the projection method, where a bistab sparse matrix solver was used to solve the pressure Poisson equation every time step 21, 23. A normal velocity component was specified as an inflow condition to the nasal cavity determining the inflow mass flow rate. Zero gradient tangential velocity gradients were also used as part of the inflow condition. Zero velocity gradients were used as outflow conditions at the outlet of the nasal cavity, which is the nostril during expiration and the pharynx during inspiration. When the external flow was also considered for expiration, a symmetry sagittal (spanwise) plane was placed at the sagittal location of the septum, modeling the effect of the other nostril, taking it as a mirror nostril. All other sides of the computational domain opened to the ambient air had zero velocity gradients as outflow conditions. 


\subsection{Experimental methodology:}

For the experimental flow visualization the PIV measurements were carried out on a 2.54:1 silicon model built using Fused Deposition Modelling (FDM) technology from the CAD file of the computational nasal cavity, see schematic diagram and picture in Figs 2. A similar scale for the experimental model was used by Brucker et $a l^{5}$ and Cozzi et al. ${ }^{26}$. An aluminum sheet was cut and added to the experimental model, to represent the face in the computational data. A plate was added at the septum location as a wall of symmetry mimicking the effect of the second nostril and assuming it is a mirror image of the experimental nostril. The entire model was then painted in mat black to avoid laser reflection during the experiments.

The airflow through the nasal cavity was designed to be kinematically similar to the computational data by matching the Reynolds number for the steady expiration phase. The air entered the nasal cavity through the nasopharynx opening, allowing comparison of expiratory flows outside the nostril at three different volume flow rates of 130, 380 and $635 \mathrm{ml} / \mathrm{s}$ which corresponded to 50,150 and $250 \mathrm{ml} / \mathrm{s}$ in the computational studies.

The PIV system comprised of a twin Nd:YAG Lasers (532 nm wavelength, 10-400 mJ/pulse at $10-30 \mathrm{~Hz}$ ). The lights from the lasers were combined and directed to the experimental set up via a beam delivery system and a combination of spherical and cylindrical lenses to expand the laser light into a plane and into a thin sheet respectively. The laser head was adjusted so that the laser sheet passes parallel through the model where it has the smallest thickness. The scattered light by the tracer particle (olive oil), which was injected to the air before entering the pharynx through a T junction, was captured using a PIVCAM 138 CCD camera. A synchronizer worked as the timing and the control module for the PIV system by connecting to the computer, camera and the lasers and synchronizing their operation. The PIV images were captured at three different planes of coronal, sagittal and parallel to the nostril plane. For the coronal plane, the nostril was divided into five equal sections for four planes and the sagittal plane cut through the nostril medially. Before capturing any image, the system was calibrated and the timing parameters were set for each flow rate. The recorded images were processed using the Insight software. This processing involved, Image conditioning, grid generation, performing the correlation (cross correlation technique), locating peaks and performing validations. 


\section{Results and analysis}

3.1 Nasal resistance and flow pattern in healthy nasal cavity

The geometry of the left-hand passage of the human nasal cavity was captured from CT (computerized tomography) scans using the Amira software for a healthy adult patient. The Amira Software has already been successfully used in various studies on bio-fluids including nasal air flow ${ }^{3,27,28}$. The iso-surface of the external enclosure of the cavity is shown in Fig 3 for the reduced grid size. Grid sensitivity was carried out for two levels of grid size; corresponding to $(129,129,65)$ and $(197,197,97)$ where the directions are in $x, y$ and $z$ respectively. This was done for several volume flow rates at inspiration and expiration, and only a small change was observed between the two grids.

The variation of the the overall flow rate with the transnasal pressure $d p$ is shown in Fig 4 , where positive flow rate corresponds to inspiration and negative to expiration, and the left and right cavities were assumed to be mirror of each others, i.e. the overall flow rate is twice the one of the left cavity. Commonly the nasal resistance (NR) is measured at $\mathrm{d} p=$ $150 \mathrm{~Pa}$, yield $\mathrm{NR}=0.22 \mathrm{~Pa} \mathrm{~s} / \mathrm{ml}$ for inspiration which is an excellent agreement for the averaged reported for the German adult population of $0.21 \mathrm{~Pa} \mathrm{~s} / \mathrm{ml}$ and well inside the ranges reported for other populations ${ }^{30}$. The much lower nasal resistance at expiration as seen in Fig 4 is also typical and was reported by others ${ }^{2}$.

To point to the cause in the NR difference between the inspiration and expiration phases, the velocity magnitude and streamlines are plotted in Figs 5 and for the flow rate of 250 $\mathrm{ml} / \mathrm{s}$ per cavity, i.e. $500 \mathrm{ml} / \mathrm{s}$ for the nose. The inspiration phase shows higher velocity magnitudes than the expiration phase but also with increased curling in the streamlines, particularly towards the upper part of the cavity as compared to the expiratory flow. This causes the air to spend more time around the turbinates and the oflaction region during inspiration than in expiration, thus supporting the conditioning of the air temperature, humidly and the oflaction sense during inspiration. These are the essential functions of the nose. The curling streamlines are due to vorticity generation around the nasal valve, taking kinetic energy away from the streamwise convection of the air towards the pharynx during inspiration. Thus higher potential energy in terms of pressure difference over the nasal cavity is needed to produce the same flow rate in inspiration as compared to expiration.

Two concentrated vortices are revealed in Figs 5, one anterior near the nasal valve and another posterior near the pharynx. In expiration the posterior vortex moves to the cavity's floor due to a steep change in flow direction. The anterior vortex moves away from the nasal valve towards the nostril as it was convected by the flow towards the nostril. 
An increase in the turbulent kinetic energy $k$ as relative to the inlet value was observed at the nasal valve and the pharynx for the inspiratory and expiratory phases respectively. However in inspiration the increase in $k$ from inlet value was of higher level and occurred at lower flow rates, e.g. it increased by $2 \%$ for inspiratory flow rate of $200 \mathrm{ml} / \mathrm{s}$ and $9 \%$ for flow rate of $300 \mathrm{ml} / \mathrm{s}$, while no increase in $k$ for expiratory flow rate of $200 \mathrm{ml} / \mathrm{s}$ and only $4 \%$ for flow rate of $300 \mathrm{ml} / \mathrm{s}$. This also supports the finding that nasal resistance and kinetic energy losses are higher in inspiration than in expiration.

\subsection{Internal nasal cavity flow with partial blockage in the airways.}

Four types of blockage were considered; (i) deviated septum (ii) enlarged inferior turbinate (iii) enlarged middle turbinate and (iv) nasal polyps. These are only some of the typical blockages and not the full range of possible blockages. Nasal polyps can appear from the ethmoid sinuses into the nasal cavity thorough the middle turbinate ${ }^{29}$. Hence only a typical blockage at the superior region of the nasal cavity was modelled. A more comprehensive survey of blockages should be considered in a future study in order to advance to higher TRLs (Technology Readinesses Levels). In all cases a generic blockage with a typical length scale was assumed. The blockages caused by the deviation in the septum are illustrated in Figs 6, where two common cases are considered; anterior deviated septum and posterior deviated septum. The corresponding contours of velocity magnitudes are given in Figs $7 \&$ 8 for inspiration and expiration of $200 \mathrm{ml} / \mathrm{s}$ (per cavity) respectively.

An increase in the inspiratory velocity magnitude is observed for both obstructed cavities at the location of the blockage due to narrowing of the nasal airways. There is also a reduction in the flow rate by almost half, through the olfactory region in both blocked cavities as compared to the healthy nasal cavity. This reduction, which is slightly higher in the cavity with the anterior blockage, supports the view that a healthy nasal cavity is designed to supply more air to the olfactory ${ }^{18}$ and septal deviation may impair this mechanism. Similar to the inspiratory phase there is also an increase in velocity magnitude in expiration where the blockages narrow the nasal airways.

An increase in nasal resistance values is observed for both blockages due to the narrowing of the airways, yielding $\mathrm{NR}=0.6$ and $0.5 \mathrm{~Pa} \mathrm{~s} / \mathrm{ml}$ for the anterior and posterior blockages respectively for the inspiratory flow rate of $200 \mathrm{ml} / \mathrm{s}$, where for healthy cavity $\mathrm{NR}=0.27 \mathrm{~Pa} \mathrm{~s} / \mathrm{ml}$. The turbulent kinetic energy $k$ also increased near the nasal valve for the anterior blockage but no increase was observed for the posterior blockage case. This can be explained by the nasal valve being a source of turbulence ${ }^{3}$ and the anterior blockage being much closer to the nasal valve than the posterior blockage. 
Enlarged inferior turbine was also investigated for anterior and posterior blockages as illustrated in Fig 9. Significant increase in the velocity magnitude is seen for both inspiration and expirations phases in Figs $10 \& 11$ respectively, due to narrowing of the airways. The anterior blockage yields the highest velocity despite having a lower degree of area blockage. In both phases the flow is deflected upwards towards the oflaction region, meaning the oflaction function is not hampered by this kind of blockage. The nasal resistance (NR) increases from $0.27 \mathrm{~Pa} \mathrm{~s} / \mathrm{ml}$ for the healthy cavity to 0.34 and $0.41 \mathrm{~Pa} \mathrm{~s} / \mathrm{ml}$ for the anterior and posterior blockage respectively at inspiratory flow rate of $200 \mathrm{ml} / \mathrm{s}$. These are smaller increases in NR than in the blockages of deviated septum. The increase in the turbulent kinetic energy $k$ was also much lower than in the deviated septum cases. This can be explained by the location of the blockages of the enlarged inferior turbine being further from the nasal valve than the blockages due to the deviated septum.

The blockages considered for enlarged middle turbinate and nasal polyps are illustrated in Figs 12. The corresponding velocity magnitude contours for the polyps blockage at the inspiratory flow rate of $200 \mathrm{ml}$ are given in Fig 13. Slight increase in the velocity magnitude is observed due to the moderate reduction in the airways' cross-section area. This led to a minimal effect on the nasal resistance and turbulent kinetic energy $k$ generation. Similar behaviour was observed for the expiration phase and the enlarged turbinate blockages, showing that such blockages have small overall effects on the nasal flow. This is because of the moderate decrease in the cross-section area in a region where the flow has space to re-adjust without a significant change.

\subsection{External airflow for normal and abnormal nasal cavity geometries}

The interest in the external airflow comes from the ambition of trying to qualify the airflow and its correlation to an abnormal geometry of the nasal cavity. Hence, this can assist in identifying deformations in the nasal anatomical structure before pursing invasive or extensive tests as CT scans. The computational domain was extended into the ambient air and in order to include the air stream emitting from the nostril during expiration. A symmetry wall was used to model the effect of the second nostril taken as a mirror image of the simulated nostril as in the experimental set up. Similar to the boundary conditions used in the nostril cross-section previously, zero velocity gradient conditions were used on the other sides of the computational domain opened to the ambient air.

The steady expiratory velocity contours at the nostril cross-section are shown in Fig 14 for a flow rate of $250 \mathrm{ml} / \mathrm{s}$ and the healthy (normal) nasal cavity. Along side the computational contours, the experimental contours are also given as were measured from 
the PIV study of the enlarged cavity model of 2.54:1. One should note that the Reynolds number based on the bulk velocity was kept the same for the computational and experimental results and thus obviously the experimental velocities are of lower magnitude than the computational velocities due to the enlarged experimental model. Furthermore, although the internal geometry of the experimental model was 2.54:1 of the computational model down to the accuracy of the 3D printer and mesh interpolation in the CFD, the cross-sections of the nostril and pharynx had to be refined manually due to manufacturing issues. Nevertheless, the flow patterns are very similar between the two results, showing the bulk of the flow at the wider section of the nasal cross-section marked by Line 2 in Fig 14.

To provide a quantitative comparison between the computational and experimental results the velocities along Lines 1 and 2 of Fig 14 were normalised by the respective computational and experimental bulk velocities and their distributions are shown in Figs 15 \& 16. The dimensionless distance forming the horizontal axis of those figures was normalised by the hydraulic diameter of the nostril cross-section area, i.e. the diameter of a circle having the same area. The forward tip of the nostril is marked as zero on the horizontal axis of Fig 15. Both computational and experimental distributions show a peak velocity at the same location, not far from the tip and followed with a long tail failing backwards. There is a very good agreement between the two lines, when considering the computational results are based on RANS and the slight discrepancy between the two nostril cross-sections as exhibited by the slightly longer computational tail.

The dimensionless velocity magnitude distribution along Line 2 is shown in Fig 16 . The septum is located at the end of the velocity distribution on the right direction of the horizontal axis. Fair to good agreement is revealed between the computational and experimental results, where both show that the peak velocity area is located near the outside part of the nostril. The computational distribution smears the peak's fluctuations seen in the experimental result which can be a result of the up-winding $2^{\text {nd }}$ order scheme, but the computational distribution also shows a mildly wider velocity distribution.

One cannot expect a patient to be assessed using a PIV system that relies laser measurements and particles being embedded in the flow. However, other visualisation techniques based on light refraction as Schlieren may be used. Such systems capture the air density variation and its double $Z$ configuration was used to produce the visualisation of the air stream at the sagittal plane coming from the first author's nostrils in Fig 17a. The air temperature as it leaves the nostrils is of the body's temperature which helps in the flow visualisation. The computational contours of the air stream velocity magnitude at flow 
rate of $200 \mathrm{ml} / \mathrm{s}$ are shown along side in Fig 17b. Good qualitative agreement is seen between the two pictures in terms of the stream angle relative to the vertical line of the face contour, despite the probable anatomical differences between the two healthy nasal cavities. It suggests a possible universal air stream angle, but this requires a further statistical study on wide range of the population.

To examine the effect of the possible partial nasal blockages discussed in Section 3.2, the corresponding computational external air streams for the expiratory flow rate of $200 \mathrm{ml} / \mathrm{s}$ are shown in Figs 18 against the air stream coming out of the nostril of the healthy (normal) cavity. The blockages due to the deviated septum show the highest visual effect on the external air stream as they did on the internal airflow in terms of nasal resistance and turbulent kinetic energy production during the inspiration phase. The anterior blockage of Fig $18 \mathrm{~b}$ causes the air stream to flow at a higher angle relative to the vertical than in the case of the healthy cavity, but also to be more concentrated and a narrower stream with higher velocity. The posterior blockages of Fig $18 \mathrm{c}$ also caused higher velocities in the external air stream, but it flows in a smaller angle as relative to the vertical and the stream is thicker than in the normal cavity case of Fig $18 \mathrm{a}$.

The behaviour of the external air stream due to the deviated septum blockages can be explained by an analogy to a water hose used to water a garden. When the gardener wants the water stream to go further s/he narrows the exit of the hose, similar to what happens with the nostril air stream and the anterior blockage of Fig 18b that is just behind the nasal valve and not far from the nostril. On the other hand the posterior blockage is far from the nostril and thus the air has the time and space to re-adjust before leaving the nostril, resulting in the thicker stream and reduced stream angle as it happens in the water hose example when narrowed somewhere well upstream of the exit. Of-course the water jet is much more affected by gravity than the nostril air stream, but both follow the same law of continuity.

As all flows in Figs 18 have the same flow rate then a blockage tends to increase the speed in the external air streams. However, the smaller or further the blockage is from the nasal valve the smaller is the effect on the external air stream. This is particularly seen in Fig $18 \mathrm{f}$ that corresponds to the partial blockage of the nasal polyp which also had a negligible effect on the overall nasal resistance during inspiration as discussed in Section 3.2. The reason is obvious as the internal flow is given opportunity to readjust itself before entering the nasal valve and then out to the nostril. Similar low effect is seen in Fig 18e due to the partial blockage caused by the enlarged middle turbinate that although it has 
an effect on the cross-section area more than the polyp it is located in the posterior area near the pharynx.

The velocity contours in Fig $18 \mathrm{~d}$ are due to the blockage in the lower airway and meatus in the anterior area caused by the enlarged inferior turbinate as seen in Fig 9. Thus it affects the external air stream similar to the anterior blockage of to the deviated septum but to less extent, i.e. not as much a narrow air stream and a lower angle as relative to the vertical. This is because although that blockage is anterior it is still farther from the nasal valve than the anterior blockage of the deviated septum.

\section{Summary}

A computational and experimental study was conducted to investigate the internal and external airflows in the human nasal cavity using an anatomical reconstructed geometry of a healthy adult and possible generic partial nasal blockages. The subject of human nasal aerodynamics has attracted significant attention in the last few decades due to the effect on the life quality of those suffering from abnormal and dysfunctional nasal cavities. The rapid development in computational fluid dynamics (CFD) capabilities in the last two decades have led to an increase in computational studies in this area, where some as related to this study were reviewed to seek insight into the development of the airflow in healthy and abnormal nasal cavities.

Most of studies looking at the effect of partial nasal blockages concentrated on the internal airflow and particularly on the inspiration phase as it affects the amount of air taken by the patient. This study actually concentrated on the expiration phase as it had a direct effect on the external air stream emitting from the nostril. The motivation was to find correlation between the external air stream pattern and abnormalities in the nasal cavity geometry and thus to explore an additional method of assessing the existence and severity of partial blockages.

An anatomically accurate geometry of the nasal cavity constructed from CT scans of a healthy adult was used to build the computational model of scale 1:1 and an enlarged experimental model of scale 2.54:1 for PIV measurements. Nasal resistance (NR) found computationally showed very good agreement with literature-reported results for the averaged NR of healthy adults. The higher NR during inspiration as compared to expiration was explained by the physiological functions of the nasal cavity, requiring the air to spend more time inside the cavity during inspiration for temperature and humidly conditioning, and for olfaction. This was achieved by the generation of additional vorticity near the nasal valve during inspiration. 
Four types of generic partial nasal blockage were considered; deviated septum, enlarged inferior turbinate, enlarged middle turbinate and polyp. The deviated septum was found to have the largest effect on NR and turbulence generation during inspiration, followed by the enlarged inferior turbinate. The deviated septum blockages also had the most obvious effects on the external air stream coming out of the nostril during expiration in terms of the external air stream's angle as relative to the vertical and thickness of the stream. This was explained by the locations of the blockages. It was concluded that generally anterior blockages had higher visible effect on the external air stream than the posterior ones. Comparing the external air stream between the computational and experimental PIV results found good qualitative agreement. Flow visualisation based on the Schlieren method indicated the possible existence of a universal angle of the external air stream, where this method of flow visualisation can be used on patients. However, more statistical studies on the variation in the population is required where methods as of statistically averaging CT scans can be of use ${ }^{31}$. Such statistical studies will advance the TRL of this approach beyond the fundamentals of this study and should be able to provide clinical instructions of the flow visualizations similar to what was performed in the lab and illustrated in Fig 17a.

\section{References:}

1. Eccles R, Nasal airflow in health and disease, Acat Otol 120(5): 580-595, 2000

2. Kelly JT, Prasad AK and Wexler AS, Detailed flow patterns in the nasal cavity, J Appl Physiol 89(1): 323-337, 2000

3. Leeden RVD, Avital EJ and Kenyon G, Nasal airflow in a realistic anatomic geometry, Direct and Large Eddy Simulation VI, Kluwer Academic Publishers: 423-430, 2005

4. Wen J., Ihthavong K, Tu J and Wang S, Numerical simulation for detailed airflow dynamics in a human nasal cavity Respiratory Physiology \& Neurobiology 161(2): 125135,2008

5. Brucker C., Park KI and Limberg W, Experimental study of velocity fields in a model of human nasal cavity by DPIV in LaserAnemometry Advances and applications: Proceedings of the 7th international conference, University of Karlsruhe: 616-626, 1997

6. Hopkins LM, Kelly JT, Wexler AS and Prasad AK, Particle image velocity measurements in complex geometries. Exp Fluids 29(1): 91-95, 2000.

7. Kim JK. Yoon JH, Kim CH, Ham TW, Shim DB and Shin HA, Particle image velocimetry measurements for the study of nasal airlfow Acta Oto- Laryngologica 126(3): 282-287, 2006.

8. Weinhold IG and Mlynski G, Numerical simulation of airflow in the human nose. Eur Arch Otorhinolaryngol 261(8): 452-455, 2004. 
9. Ishikawa S, Nakayama T, Watanabe M and Matsuzawa T, Visualization of flow resistance in physiological nasal respiration: analysis of velocities using numerical simulation. Arch Otolaryngol Head Nech Surg 132(11): 1203-1209, 2006.

10. Ishikawa S, Nakayama T, Watanabe $M$ and Matsuzawa $T$, Flow mechanisms in the human olfactory groove. Arch Otolaryngol Head Nech Surg 135(2): 156-162, 2009.

11. Horschler I., Brucker $\mathrm{CH}$, Schroder $\mathrm{W}$ and Meinke $\mathrm{M}$, Investigation of the impact of the geometry on the nose flow Euro J Mech B/Fluids 25: 471-490, 2006.

12. Wang SM, Inthavong $\mathrm{K}$, Wen J, Tu JY and Xue $\mathrm{CL}_{\text {, }}$, Comparison of micron -and nanoparticle deposition patterns in a realistic human nasal cavity Respiratory Physiology \& Neurobiology 166(2) 142-151, 2009.

13. Grant O, Wafterson J, Cole J, Currie T and Hormhill D, Validation of a numerical method for assessment of human nasal airflow, AIAA 2005-5000 35th AIAA fluid dynamics conference Toronto, 2005

14. Kleven M, Melaaen MC, Djupesland PG, Computational fluid dynamics (CFD) applied in the drug delivery design process to the nasal passages: A review, J Mech Med Biol 12: $1230002,2012$.

15. Faramarzi MF, Baradaranfar MH, Abouali O, Atighechi S, Ahmadi G, Farhadi P, Keshavarzian E, Behniafard N and Baradaranfar A, Numerical investigation of the flow field in realistic nasal perforation geometry, Allergy \& Rhinology 5(2): 70-e77, 2014.

16. Nomura T, Ushlo M, Kondo $K$ and Yamasoba, Effect of nasal septum perforation repair surgery on three-dimensional airflow: an evaluation using computational fluid dynamics, Eur Arch Otorhinolaryngol, 272: 3327-3333, 2015.

17. Patel RG, Garcia GJM, Frank-Ito DO, Kimbell JS and Rhee JSR, Simulating the nasal cycle with computational fluid dynamics Otolaryngol Head Nech Surg 152(2): 353-360, 2015.

18. Kim SK, Heo CE, Seo A, Na Y, S.K. Chung, Correlation between nasal airflow characteristics and clinical relevance of nasal septal deviation to nasal airway obstruction, Respiratory Physiology \& Neurobiology 192: 95-101, 2014

19. Chen $X B$, Lee HD, Chong VF and Wang $d Y$, Impact of interior turbinate hypertrophy on the aerodynamics pattern and physiological function of the turbulent flow - a CFD simulation model, Rhinology 48(2): 163-168, 2010.

20. Grutzenmacher S, Robinson DMB, Lang CA, Lebe EA, Knape UC and Mlynski G, Investigations of the influence of external nose deformations on nasal airflow $O R L$ 67(3): 154-159, 2005.

21. Suponitsky V, Avital E, Gaster M, On three-dimensionality and control of incompressible cavity flow, Phys Fluids 17(10): 104103, 2005

22. Dabnichki $P$ and Avital $E$, Influence of the position of crew members on aerodynamics performance of two-man bobsleigh, J Biomech 39(15): 2733-2742, 2006 
23. Singh KM, Avital EJ, Williams JJR, Ji C, Bai X and Munjiza A, On parallel pre-conditioners for pressure Poisson equation in LES of complex geometry flow, Int J Num Meth Fluids 83(5): 446-464, 2017

24. Quadrio M, Pipolo C, Corti S, Lenzi R, Messina F, Pesci C and Felisati G, Review of computational fluid dynamics in the assessment of nasal air flow and analysis of its limitations, Eur Arch Otorhinolarygol 271: 2349-2365, 2014

25. Hosseini G, Ji C, Xu D, Rezaienia MA, Avital E, Munjiza A, Williams JJR, Green JSA, A computational model of ureteral peristalsis and an investigation into ureteral reflux, Biomedical Eng Lett: 1-9, 2017

26. Cozzi F, Felisati G and Quadrio M, Velocity measurement in nasal cavities by means of sterioscopic PIV - Preliminary tests, J Phys: Conf Ser 882: 012010, 2017

27. Zhao K, Scherer PW, Hajiloo SA and Dalton P, Effect of anatomy on human nasal air flow and odorant transport implications for olfaction, Chem Senes 29: 365-379, 2004

28. Chen J, Smith AD, Kahn MA, Sinning AR, Conway ML and Cui D, visualization of stereoscopic anatomic models of the paranasal sinuses and cervical vertebare from the surgical and procedural perspective, Anat Sci Educ 10: 598-606, 2017

29. Ludman $\mathrm{H}$ and Bradley $\mathrm{PJ}, \mathrm{ABC}$ of ear, nose and throat $5^{\text {th }}$ ed, Blackwell Publishers London, 2007

30. Gupta S, Bansal A, Rammani S, Kumar S and Goyal NK, Assessment of nasal airflow resistance in the healthy population of Chattisgarh by active anterior rhinomanometry, Indian J Otolaryngol Head Neck Surg 64(4): 338-340, 2012

31. Nejati A, Kabaliuk N, Jermy MC and Cater JE, A deformable template method for describing and averaging the anatomical variation of the human nasal cavity, BMC Medical Imaging 16:55, 2016 


\section{List of Figures:}

Figures 1: Schematic description of the nasal cavity's typical (a) sagittal cross-section and (b) coronal middle cross-section adapted from Leeden et al. ${ }^{3}$.

Figures 2: (a) Schematic diagram of the PIV apparatus, where a plate (not shown) was added on the side of the model as a symmetry wall to mimic the effect of the other nostril, and (b) a zoomed picture on the inner cavity of the model.

Figure 3: Sagittal view of the modelled nasal cavity, indicating six sections of A: Nasal vestibule, B: Nasal valve, C: Anterior region of the main nasal airway, D: middle section of the main nasal airway, E: Posterior region of the main nasal airway and F: nasopharynx.

Figure 4: Overall flow rate (sum of left and right cavities) variation with the transnasal pressure.

Figures 5: Velocity magnitude and streamlines at sagittal plane for (a) inspiration and (b) expiration at the flow rate of $250 \mathrm{ml} / \mathrm{s}$, where the arrows point to two vortices, anterior vortex (left arrow) and another near the pharynx (right arrow).

Figure 6: Nasal airways that are plotted for healthy nasal cavity (Top), cavity with anterior deviated septum (Middle: blocking the anterior region marked by the red arrow) and the cavity with deviated septum blocking the posterior part (Bottom: Marked by the red arrow). The cross-sections correspond to Fig 3.

Figure 7: Inspiratory velocity magnitude contours at flow rate of $200 \mathrm{ml} / \mathrm{s}$, where the top, middle and bottom plots correspond to the conditions described in Figure 6.

Figure 8: Expiratory velocity magnitude contours at flow rate of $200 \mathrm{ml} / \mathrm{s}$, where the top, middle and bottom plots correspond to the conditions described in Figure 6.

Figures 9: Nasal airways that are plotted for modelled enlarged inferior turbinate, partially blocking the lower airway and the meatus in the anterior region of the main nasal airway (Left: Marked by arrow) and blocking the entire lower airway and the meatus in the posterior region of the main nasal airway (Right: Marked by arrow)

Figures 10: Velocity Magnitudes for the nasal cavity free from obstruction (Top), cavity with an enlarged turbinate partially blocking the lower airway and lower meatus in the anterior region (Middle) and the cavity with an enlarged inferior turbinate in the posterior region of the main nasal airway, blocking the entire lower airway and meatus (Bottom) at inspiratory flow rate of $200 \mathrm{ml} / \mathrm{s}$.

Figures 11: Expiration velocity magnitudes at flow rate of $200 \mathrm{ml} / \mathrm{s}$ where the plots correspond to the same nasal blockage conditions as in Figs 10.

Figure 12: The nasal airways for a cavity with (a) enlarged middle turbinate blocking parts of the middle meatus at the posterior region of the turbinated area as marked by the arrow, and (b) a nasal polyp at the superior region of the turbinated area as marked by the arrow. 
Figures 13: Velocity magnitude contours for the normal (Top) and blocked cavity due to the nasal polyps (Bottom) at the inspiration flow rate of $200 \mathrm{ml} / \mathrm{s}$.

Figure 14: Computational expiatory velocity magnitude field (Left) and the experimental (Right) expiatory velocity magnitude flow field taken from the enlarged (2.54:1) nasal cavity model, at he nostril cross section for flow rate of $250 \mathrm{ml} / \mathrm{s}$.

Figure 15: Non dimensional computational (solid) and experimental (dashed) velocity magnitude profiles along Line 1 at the nostril plane of Fig 14 for the expiratory flow rate of $250 \mathrm{ml} / \mathrm{s}$.

Figure 16: Non dimensional computational (solid) and experimental (dashed) velocity magnitude profiles along Line 2 at the nostril plane of Fig 14 for the expiratory flow rate of $250 \mathrm{ml} / \mathrm{s}$.

Figures 17: External (a) experimental (Schlieren) and (b)-computational airflow pattern for the expiratory flow rate of $200 \mathrm{ml} / \mathrm{s}$ at the sagittal plane.

Figures 18: Expiratory computational external and internal nasal airflow at the sagittal median plane for the cavities; (a) healthy, (b) with anterior deviated septum blockage, (c) with posterior deviated septum blockage, (d) with enlarged inferior turbinate blocking the entire lower airway and meatus at the posterior region, (e) with enlarged middle turbinate blockage and (f) with nasal polyp blockage, where the flow rate is $200 \mathrm{ml} / \mathrm{s}$. 
a)

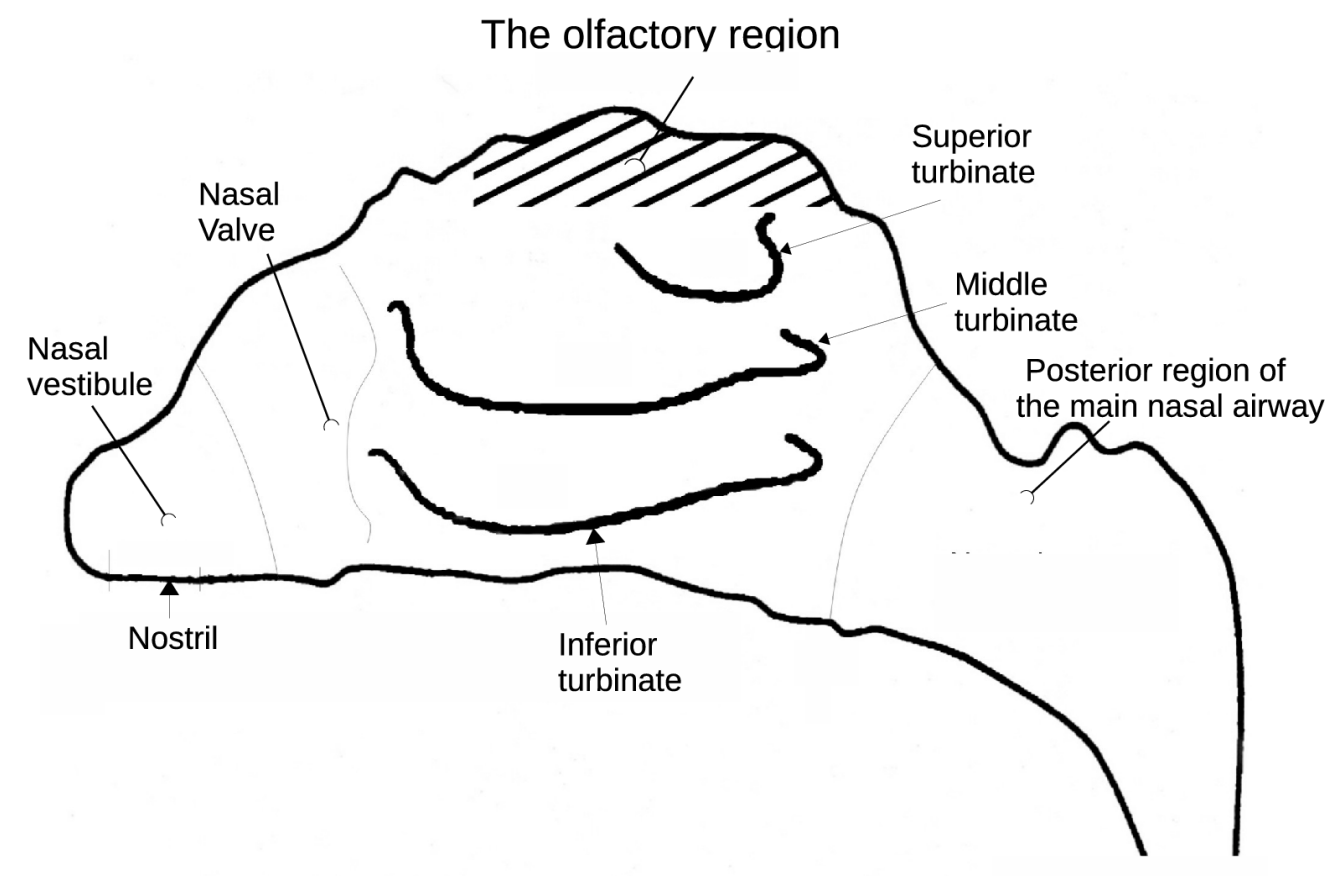

Nasopharynx

b)

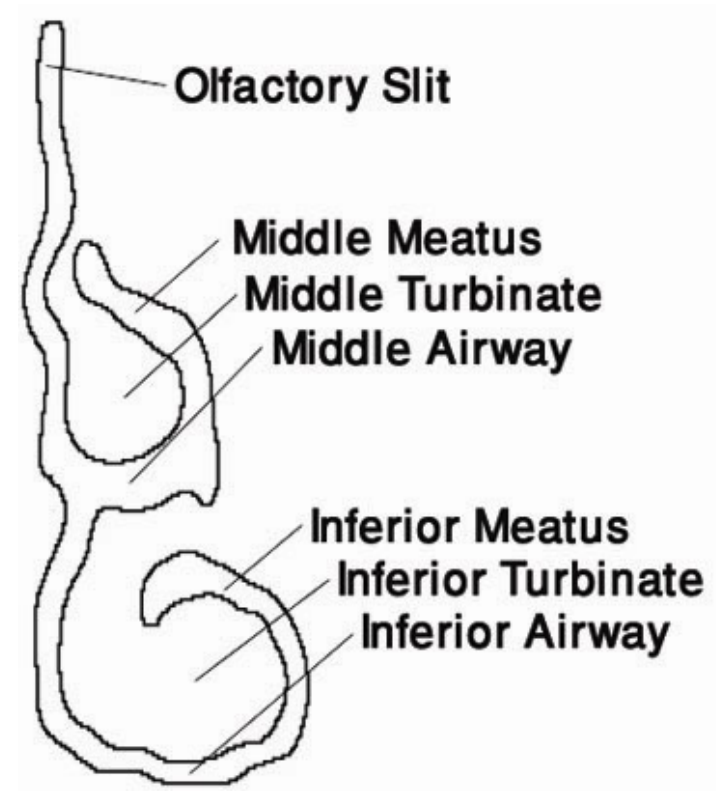

Figures 1: Schematic description of the nasal cavity's typical (a) sagittal cross-section and (b) coronal middle cross-section adapted from Leeden et al. ${ }^{3}$. 
(a)

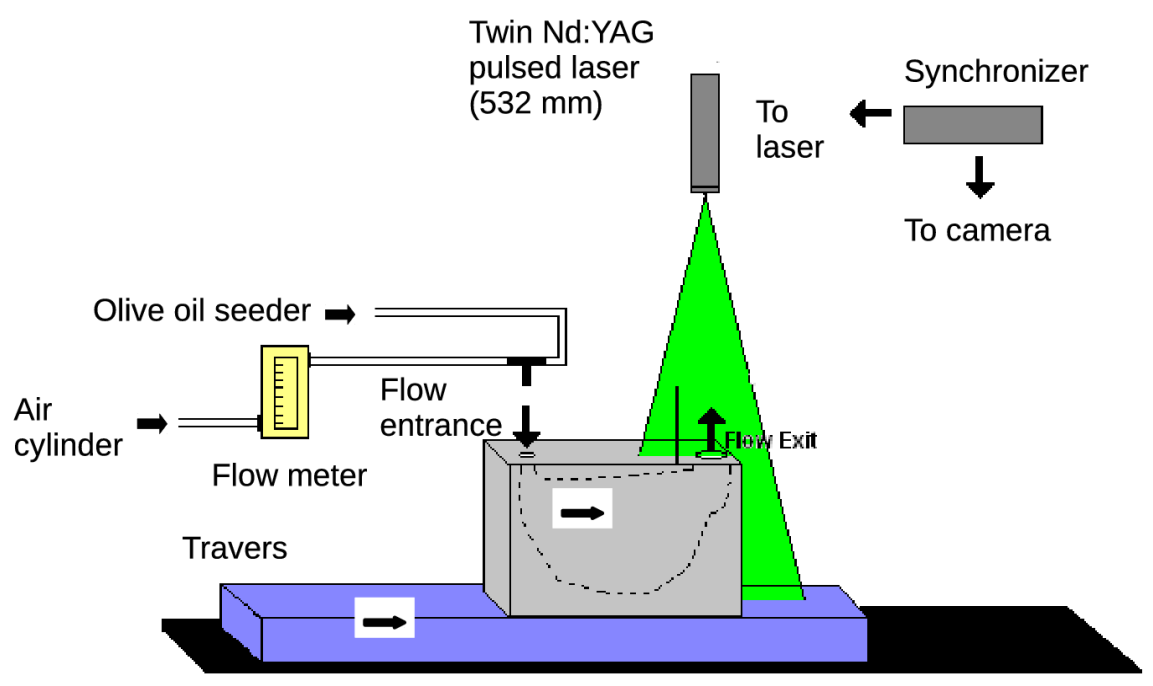

(b)

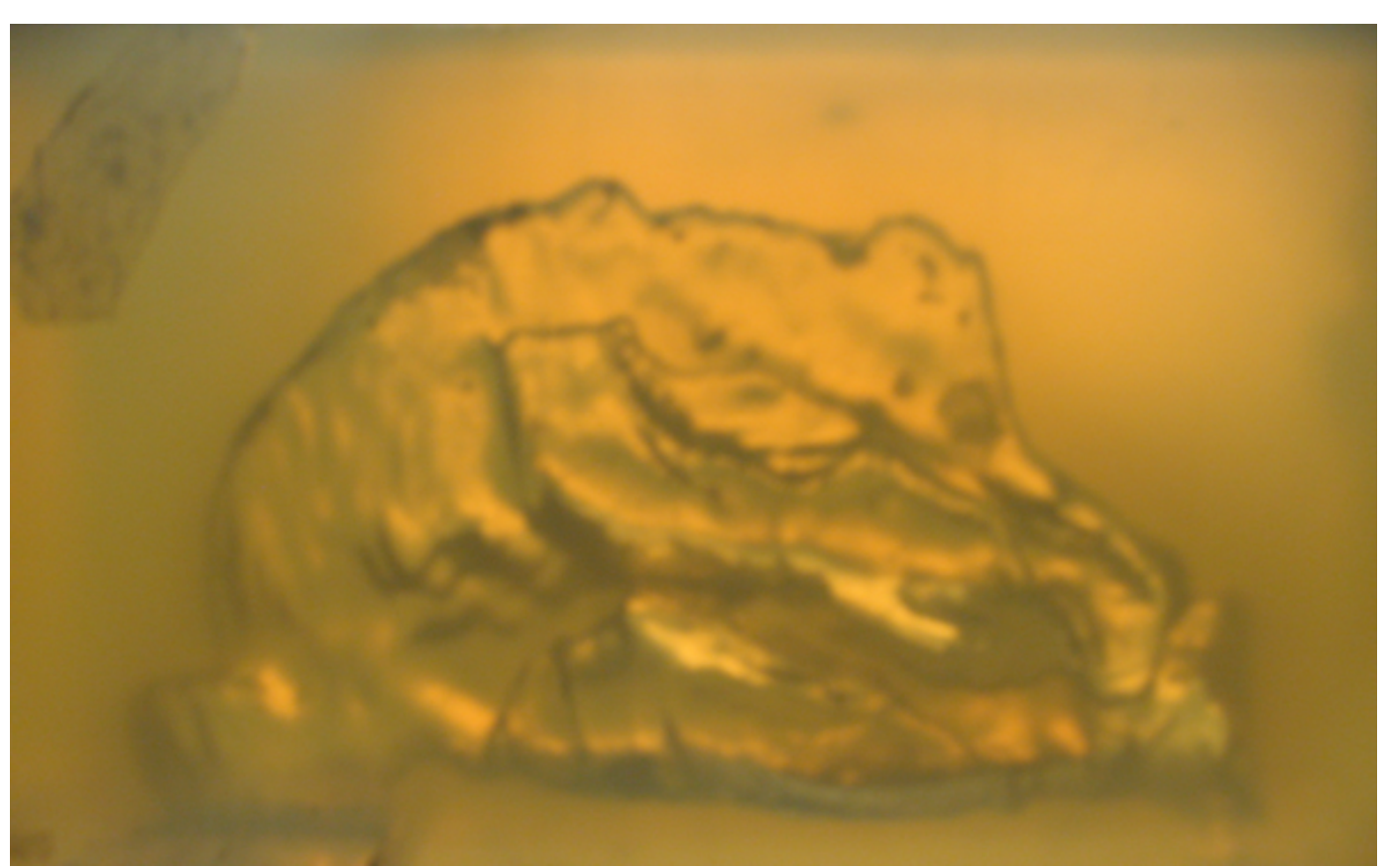

Figures 2: (a) Schematic diagram of the PIV apparatus, where a plate (not shown) was added on the side of the model as a symmetry wall to mimic the effect of the other nostril, and (b) a zoomed picture on the inner cavity of the model. 


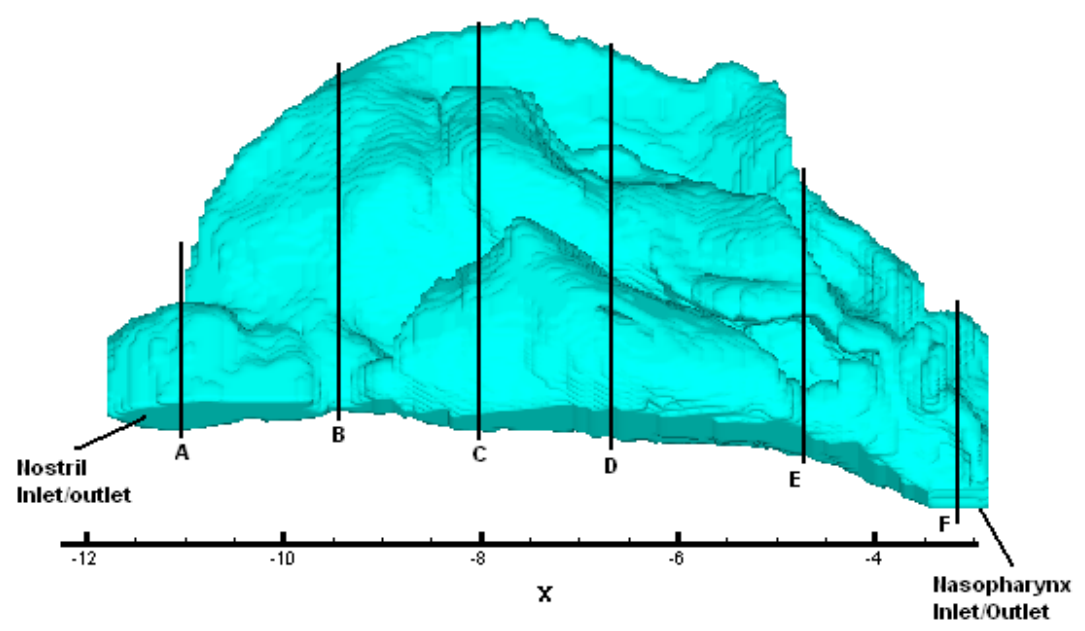

Figure 3: Sagittal view of the modelled nasal cavity, indicating six sections of A: Nasal vestibule, B: Nasal valve, C: Anterior region of the main nasal airway, D: middle section of the main nasal airway, E: Posterior region of the main nasal airway and F: nasopharynx.

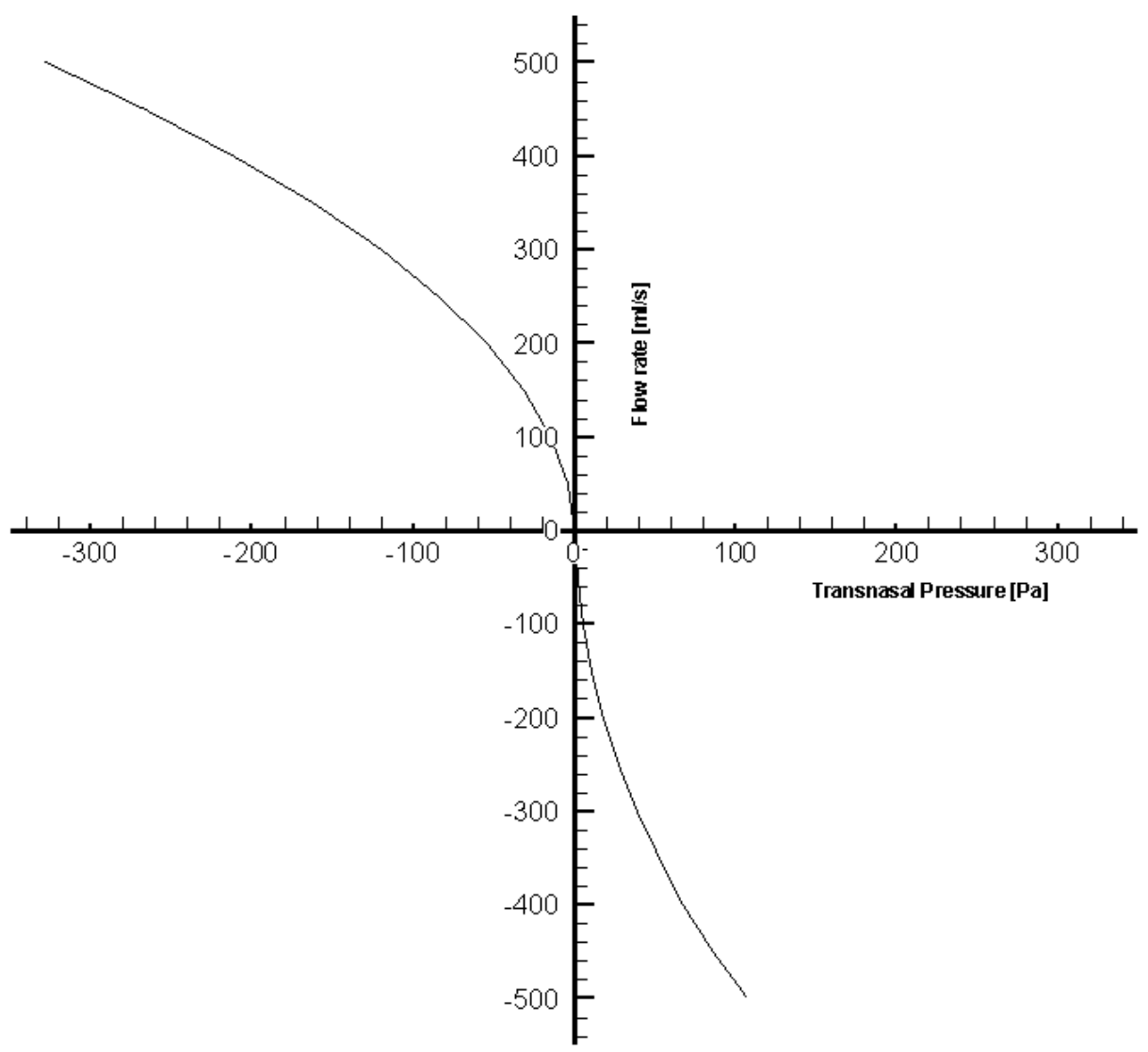

Figure 4: Overall flow rate (sum of left and right cavities) variation with the transnasal pressure. 
a)
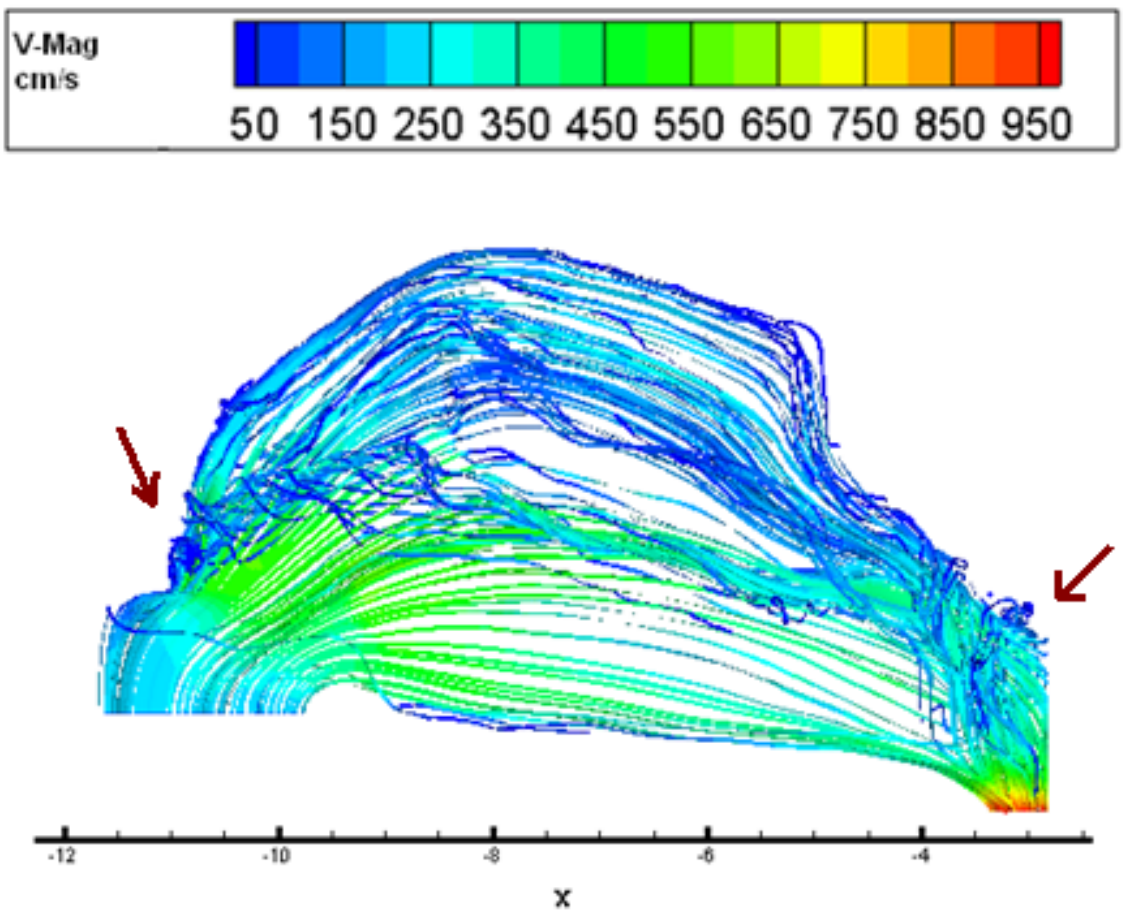

(b)
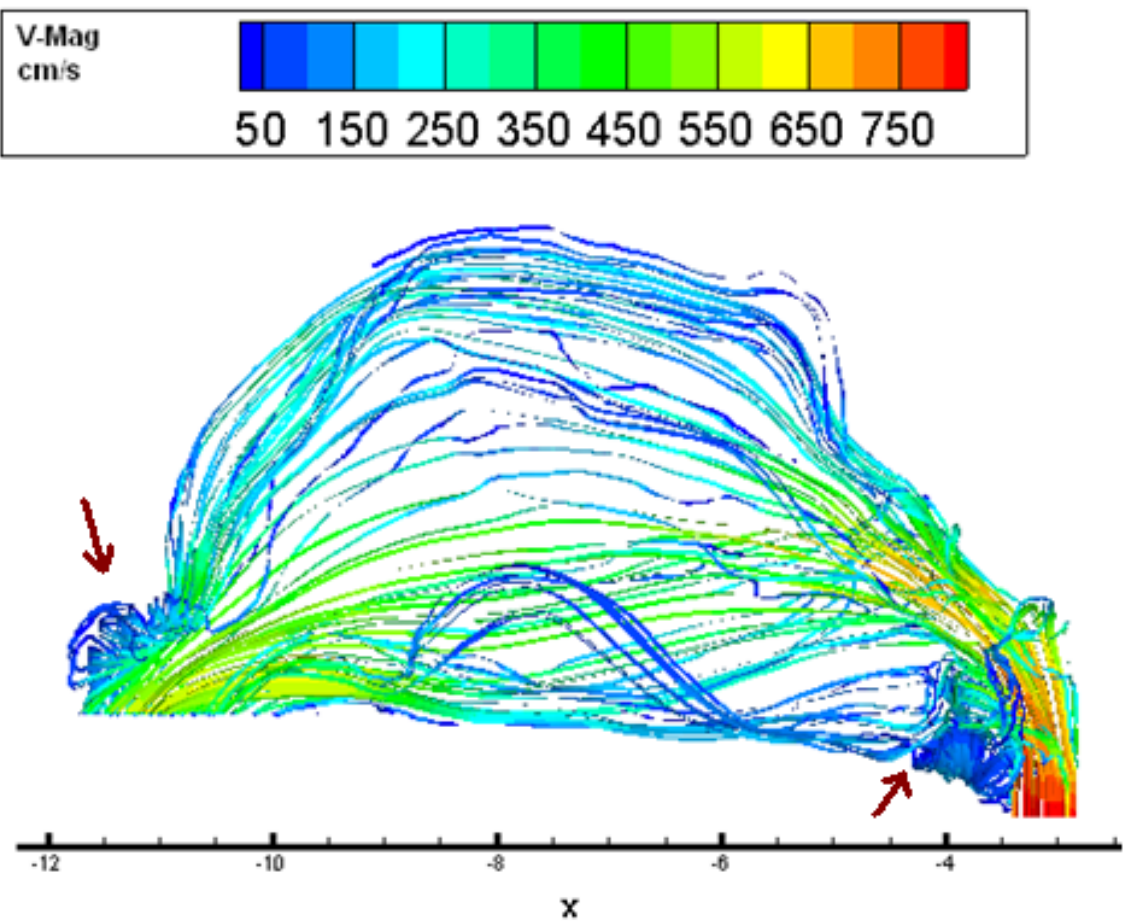

Figures 5: Velocity magnitude and streamlines at sagittal plane for (a) inspiration and (b) expiration at the flow rate of $250 \mathrm{ml} / \mathrm{s}$, where the arrows point to two vortices, anterior vortex (left arrow) and another near the pharynx (right arrow). 

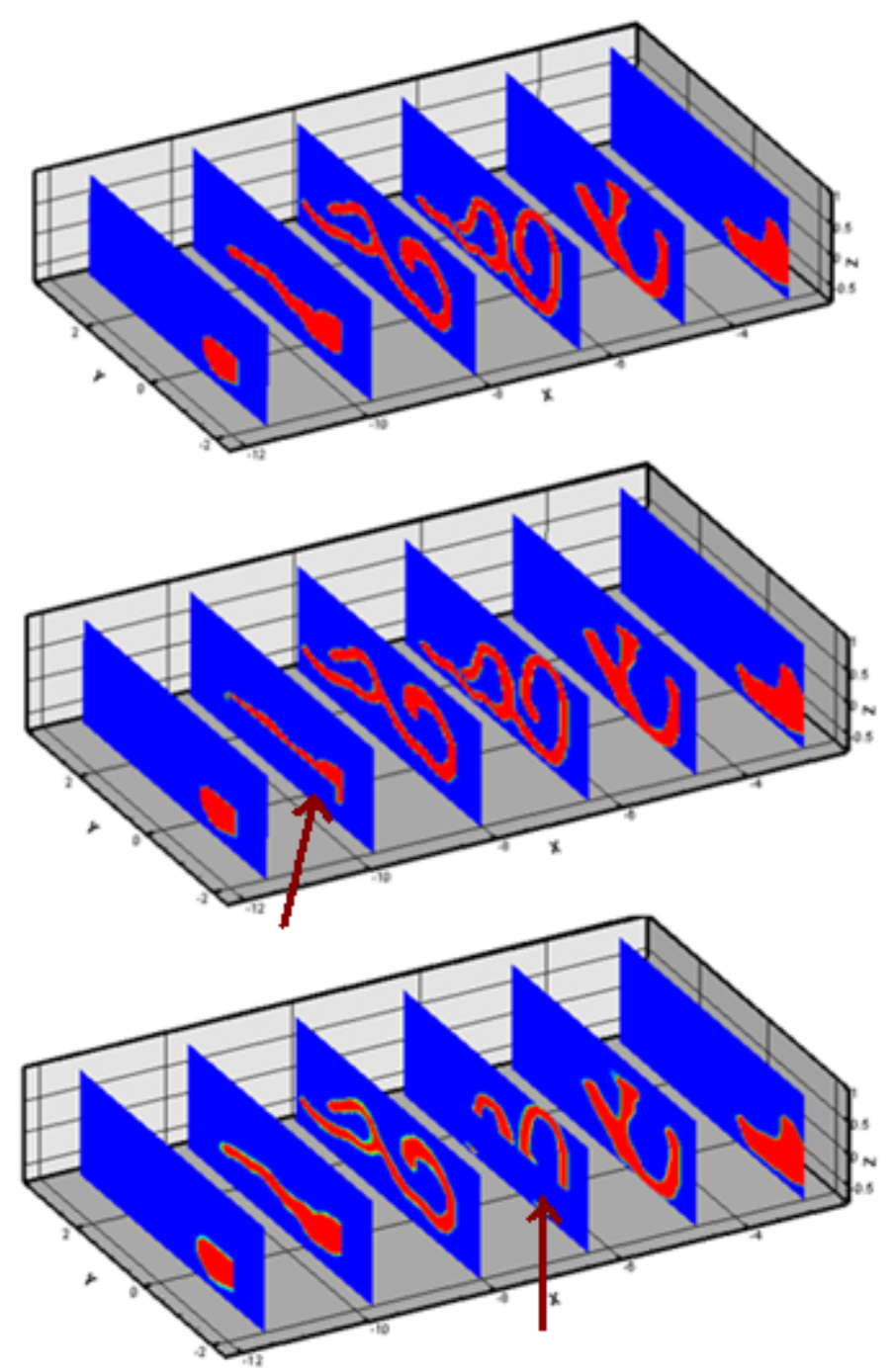

Figure 6: Nasal airways that are plotted for healthy nasal cavity (Top), cavity with anterior deviated septum (Middle: blocking the anterior region marked by the red arrow) and the cavity with deviated septum blocking the posterior part (Bottom: Marked by the red arrow). The cross-sections correspond to Fig 3. 

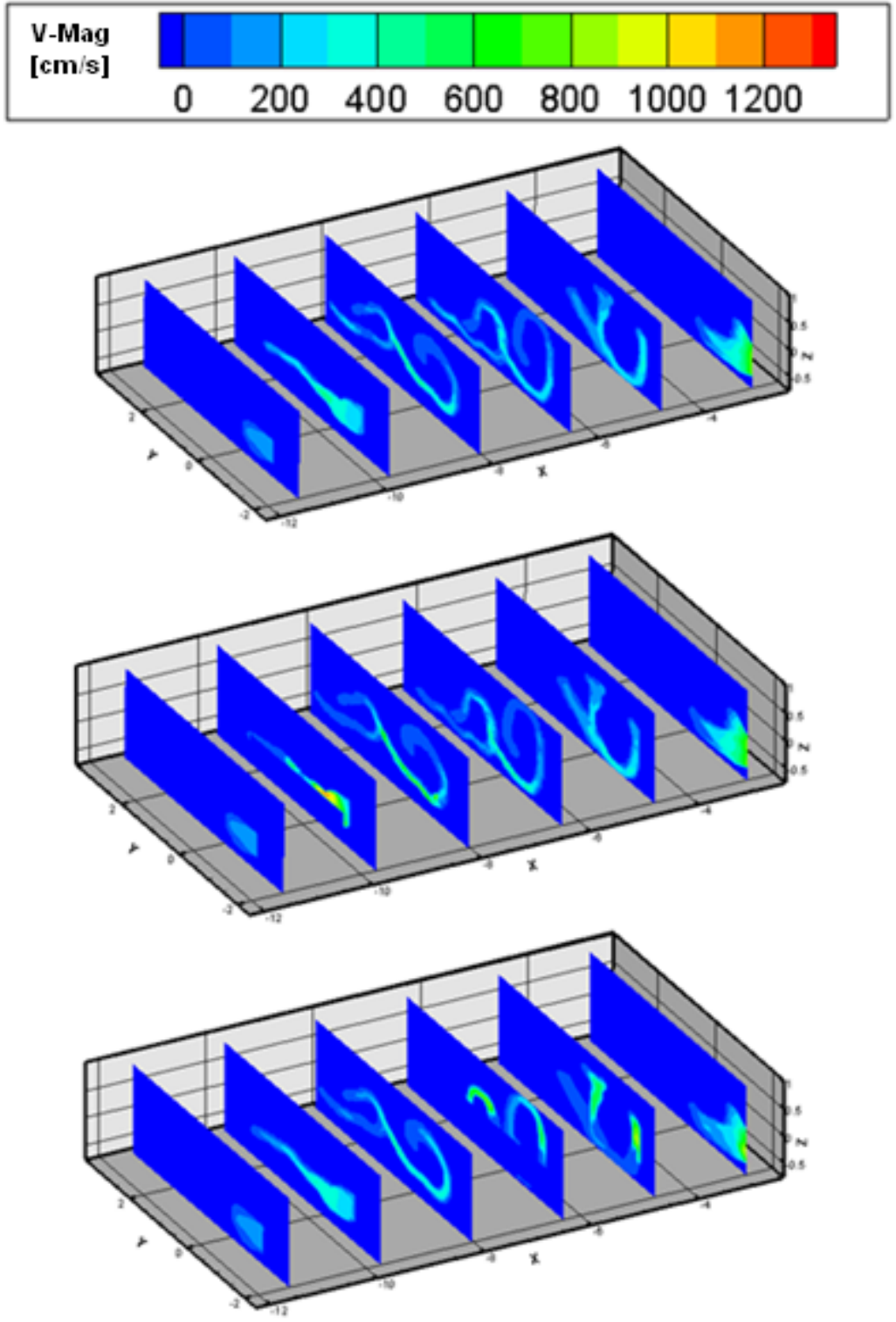

Figure 7: Inspiratory velocity magnitude contours at flow rate of $200 \mathrm{ml} / \mathrm{s}$, where the top, middle and bottom plots correspond to the conditions described in Figure 6. 

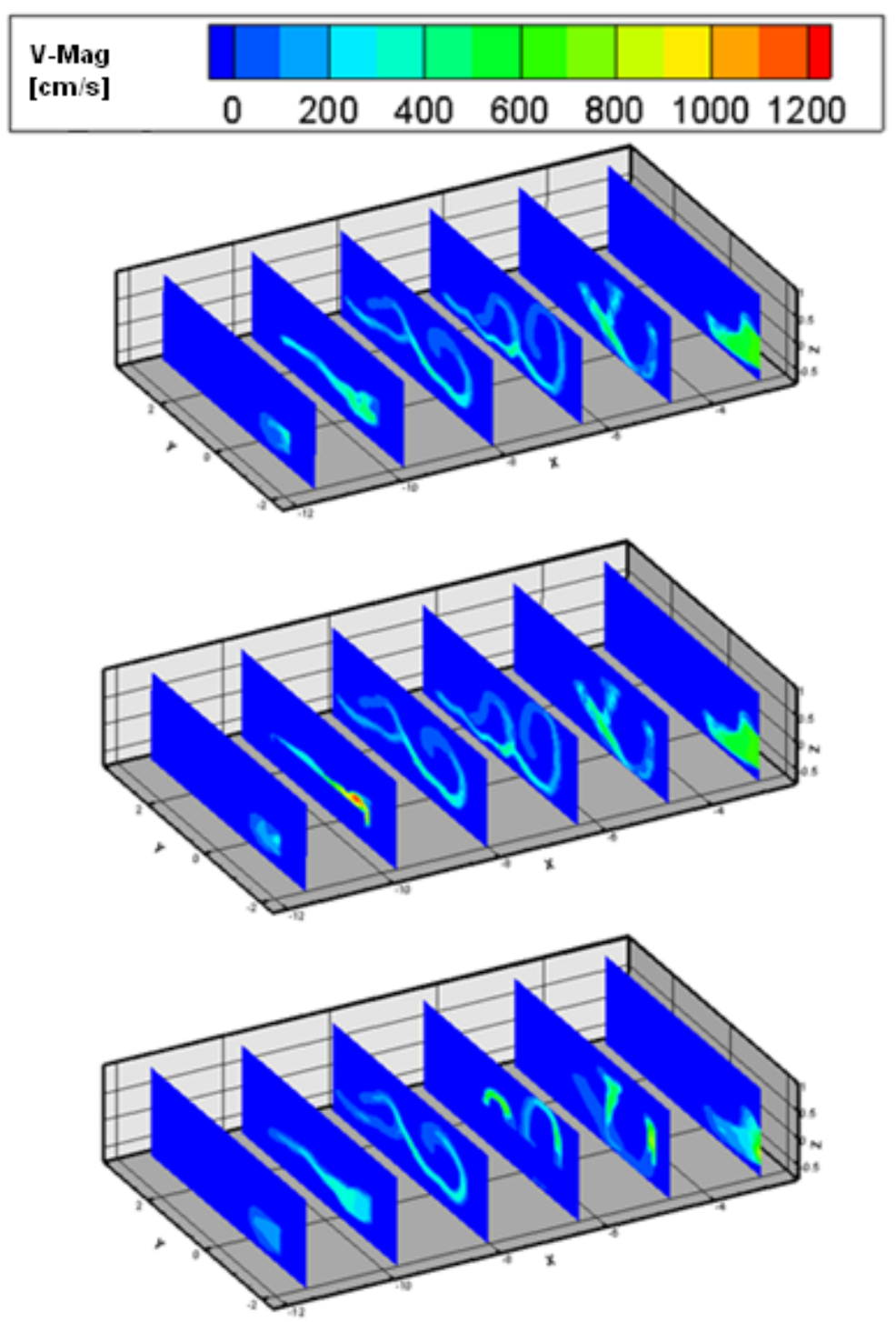

Figure 8: Expiratory velocity magnitude contours at flow rate of $200 \mathrm{ml} / \mathrm{s}$, where the top, middle and bottom plots correspond to the conditions described in Figure 6.
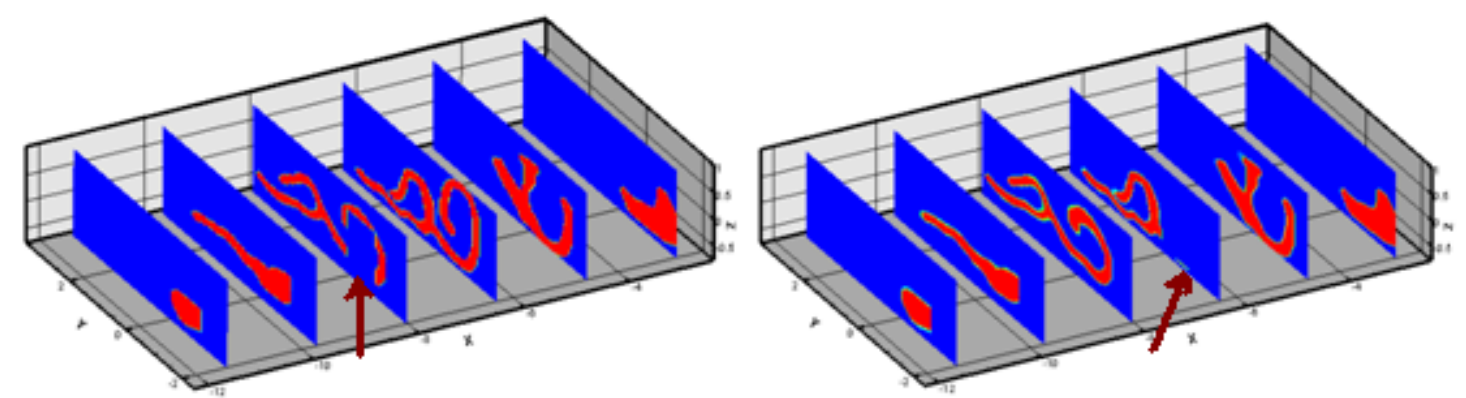

Figures 9: Nasal airways that are plotted for modelled enlarged inferior turbinate, partially blocking the lower airway and the meatus in the anterior region of the main nasal airway (Left: Marked by arrow) and blocking the entire lower airway and the meatus in the posterior region of the main nasal airway (Right: Marked by arrow). 

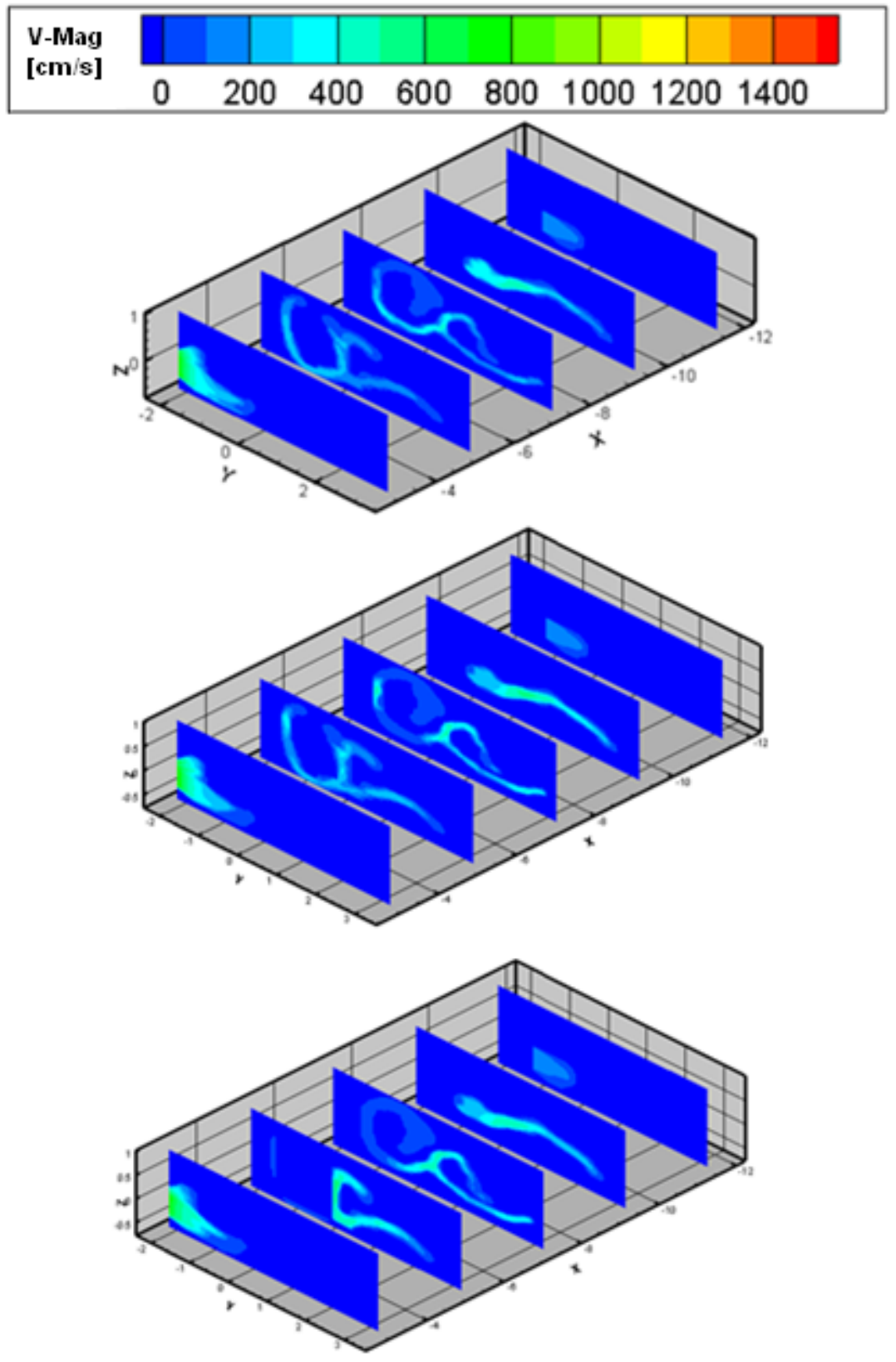

Figures 10: Velocity Magnitudes for the nasal cavity free from obstruction (Top), cavity with an enlarged turbinate partially blocking the lower airway and lower meatus in the anterior region (Middle) and the cavity with an enlarged inferior turbinate in the posterior region of the main nasal airway, blocking the entire lower airway and meatus (Bottom) at inspiratory flow rate of $200 \mathrm{ml} / \mathrm{s}$. 

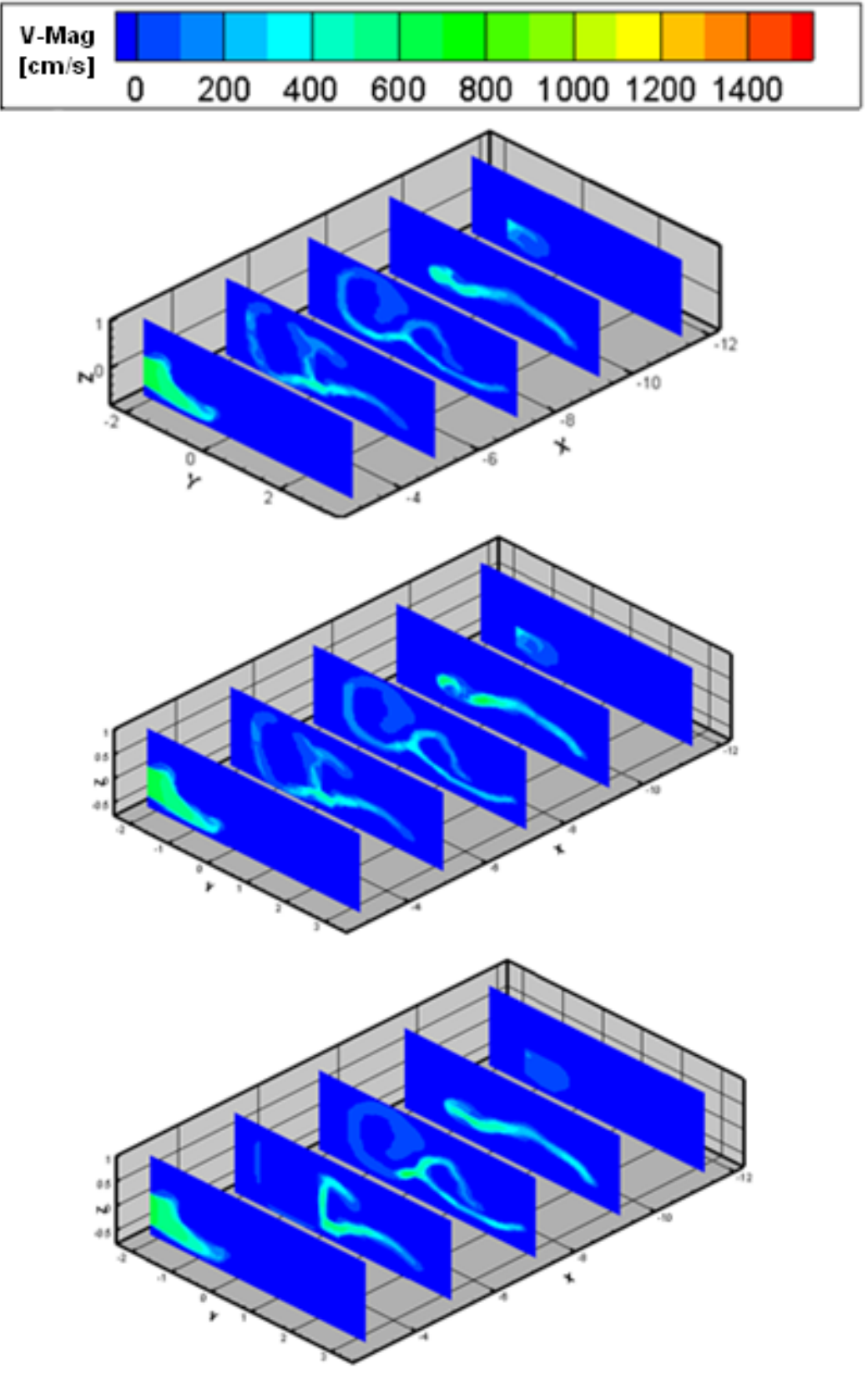

Figures 11: Expiration velocity magnitudes at flow rate of $200 \mathrm{ml} / \mathrm{s}$ where the plots correspond to the same nasal blockage conditions as in Figs 10. 
(a)

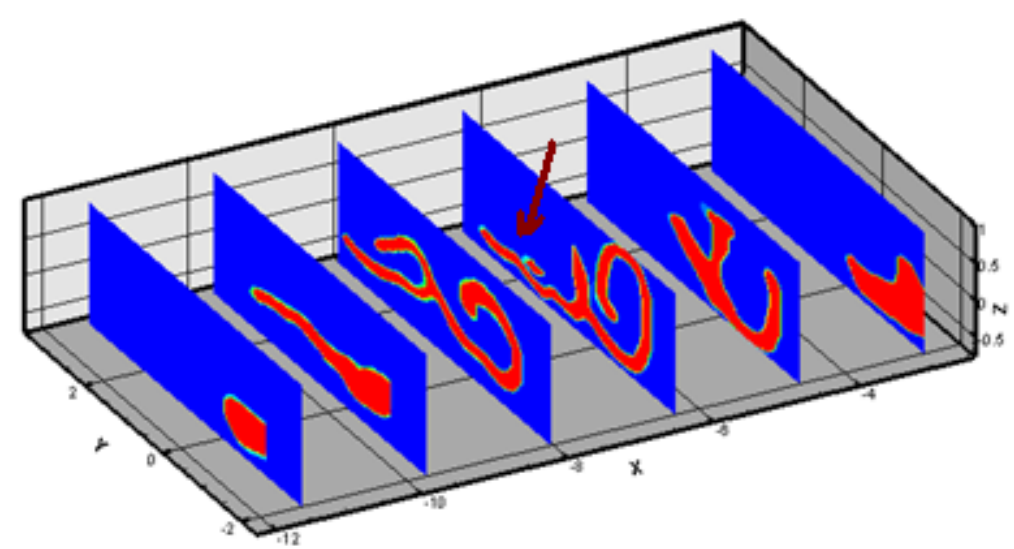

(b)

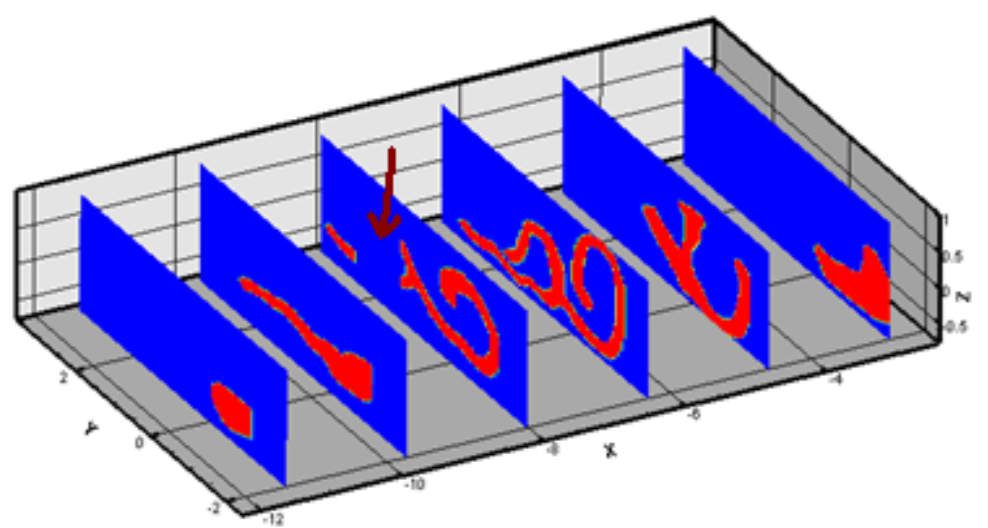

Figure 12: The nasal airways for a cavity with (a) enlarged middle turbinate blocking parts of the middle meatus at the posterior region of the turbinated area as marked by the arrow, and (b) a nasal polyp at the superior region of the turbinated area as marked by the arrow. 

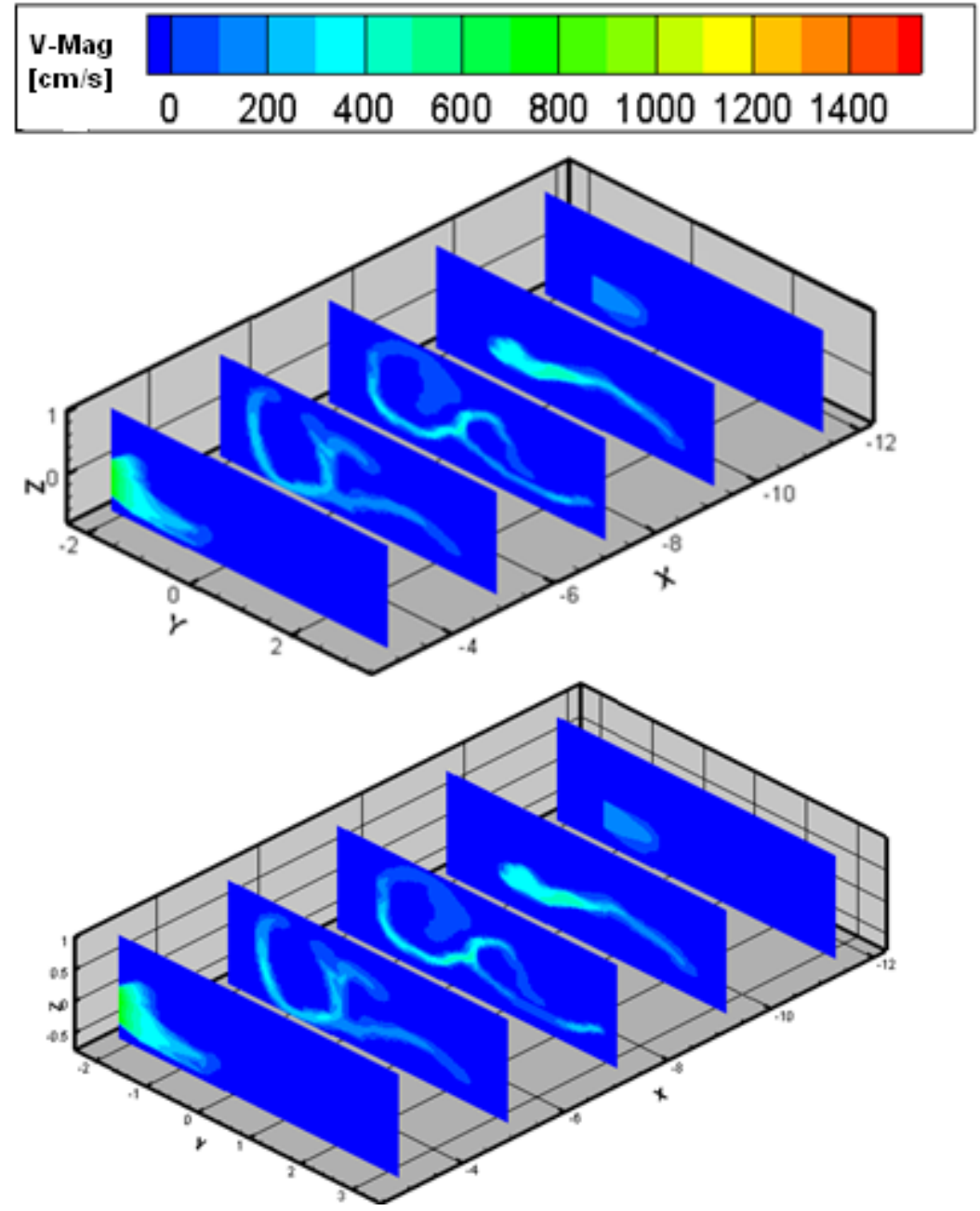

Figures 13: Velocity magnitude contours for the normal (Top) and blocked cavity due to the nasal polyps (Bottom) at the inspiration flow rate of $200 \mathrm{ml} / \mathrm{s}$. 


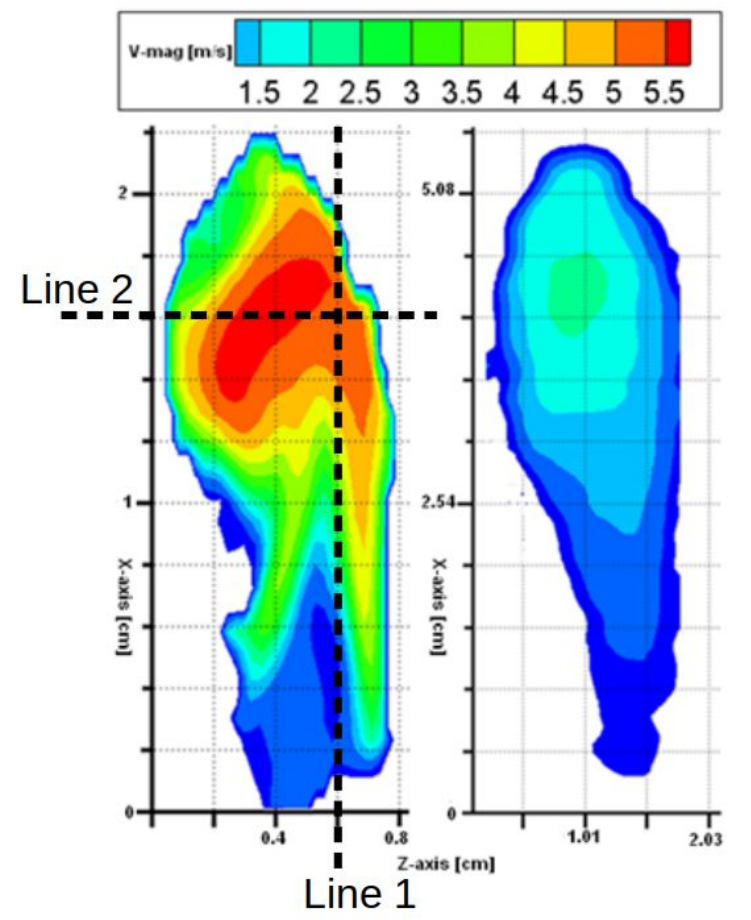

Figure 14: Computational expiatory velocity magnitude field (Left) and the experimental (Right) expiatory velocity magnitude flow field taken from the enlarged (2.54:1) nasal cavity model, at he nostril cross section for flow rate of $250 \mathrm{ml} / \mathrm{s}$.

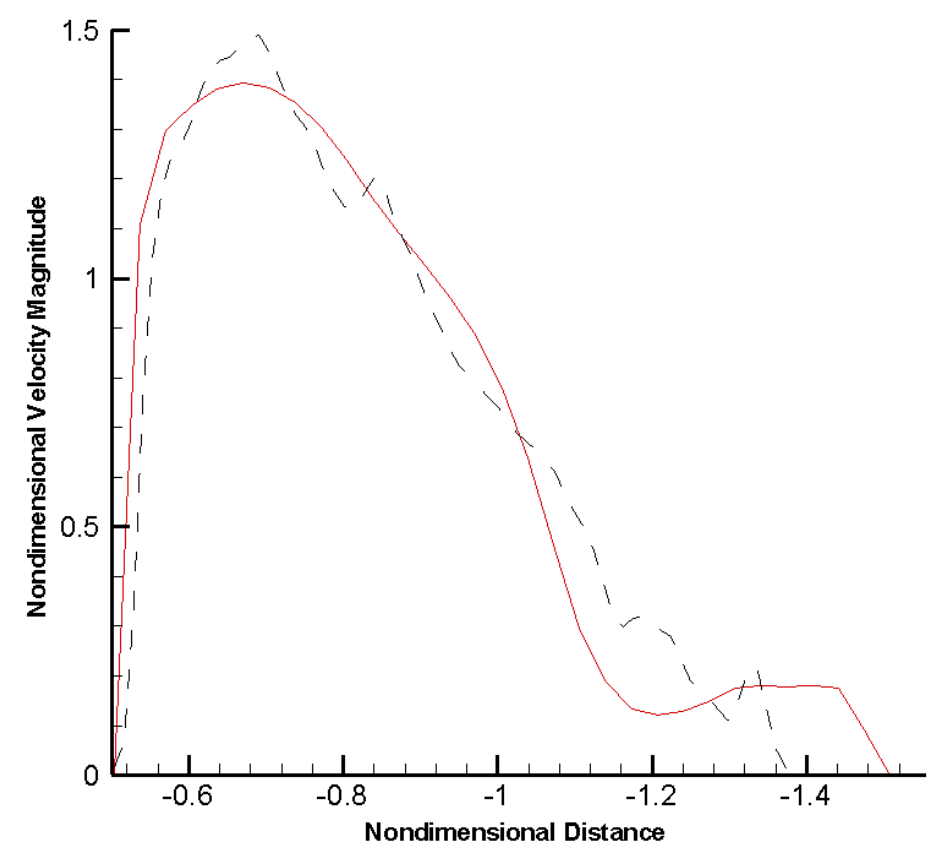

Figure 15: Non dimensional computational (solid) and experimental (dashed) velocity magnitude profiles along Line 1 at the nostril plane of Fig 14 for the expiratory flow rate of $250 \mathrm{ml} / \mathrm{s}$. 


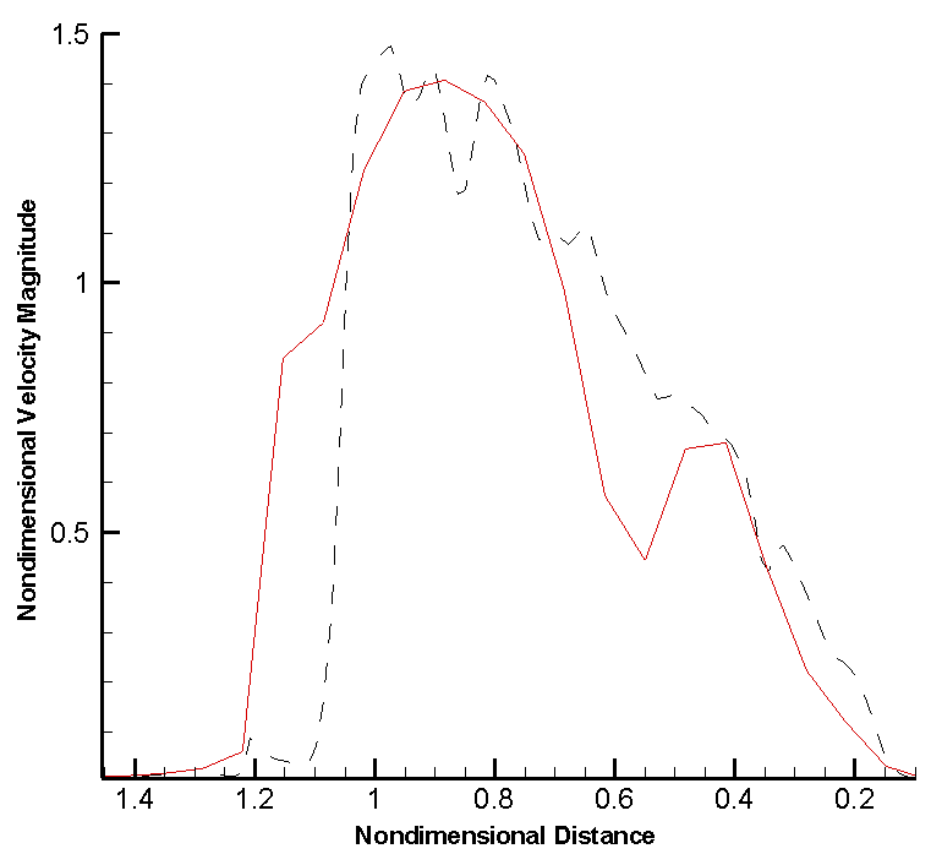

Figure 16: Non dimensional computational (solid) and experimental (dashed) velocity magnitude profiles along Line 2 at the nostril plane of Fig 14 for the expiratory flow rate of $250 \mathrm{ml} / \mathrm{s}$.

(a)

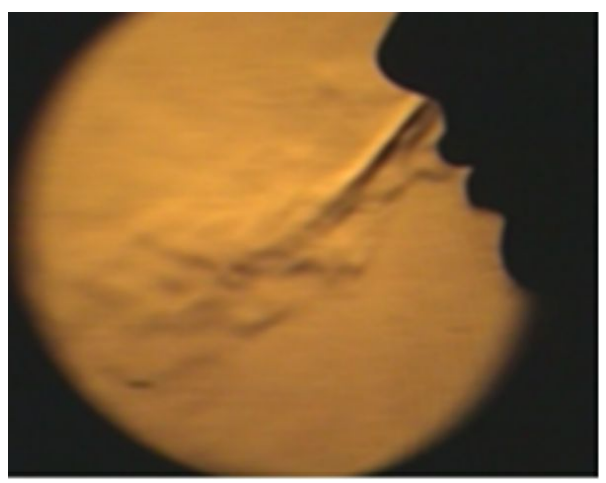

(b)

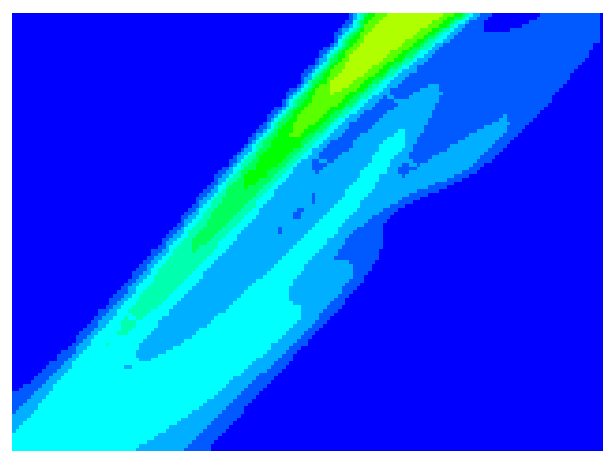

Figures 17: External (a) experimental (Schlieren) and (b)-computational airflow pattern for the expiratory flow rate of $200 \mathrm{ml} / \mathrm{s}$ at the sagittal plane. 


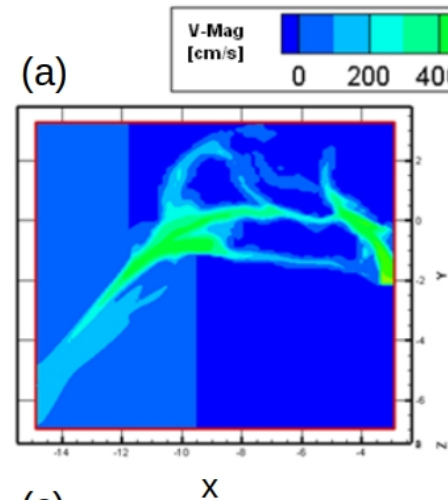

(c)

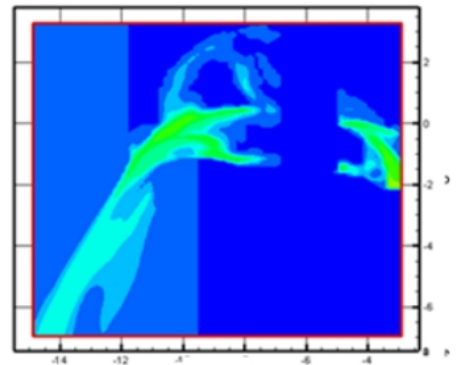

$\mathrm{X}$

(e)

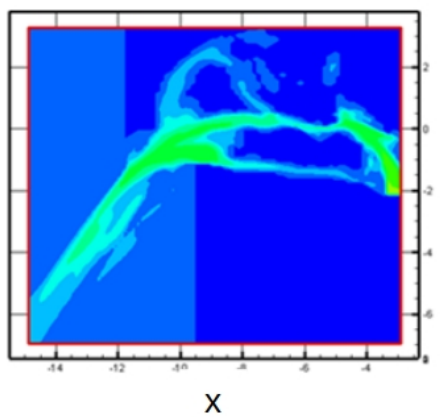

(b)

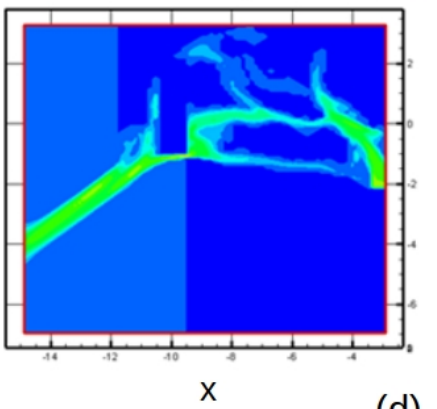

(d)

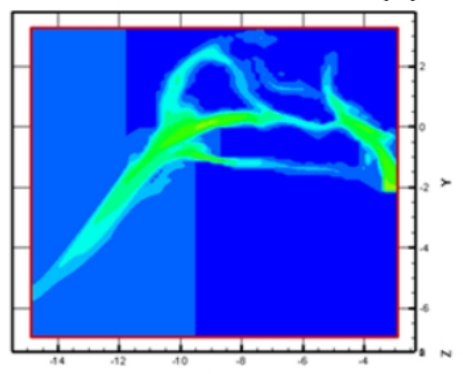

$\mathrm{X}$

(f)

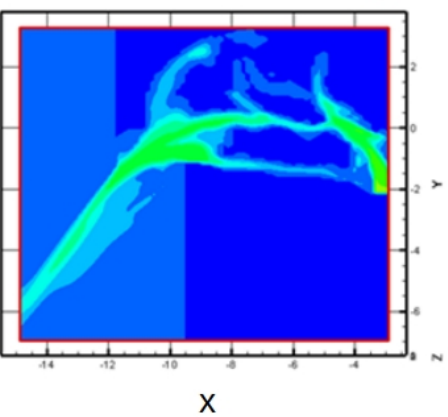

Figures 18: Expiratory computational external and internal nasal airflow at the sagittal median plane for the cavities; (a) healthy, (b) with anterior deviated septum blockage, (c) with posterior deviated septum blockage, (d) with enlarged inferior turbinate blocking the entire lower airway and meatus at the posterior region, (e) with enlarged middle turbinate blockage and (f) with nasal polyp blockage, where the flow rate is $200 \mathrm{ml} / \mathrm{s}$. 